\title{
Certain non-linear differential polynomials sharing a non zero polynomial
}

\author{
Sujoy Majumder \\ Department of Mathematics, \\ Katwa College, Katwa, \\ West Bengal-713130, India \\ email: sujoy.katwa@gmail.com, \\ sr.koshigram@gmail.com, \\ smajumder05@yahoo.in
}

\begin{abstract}
In this paper with the notion of weighted sharing of values we study the uniqueness of nonlinear differential polynomials of meromorphic functions sharing a nonzero polynomial and obtain two results which improves and generalizes the results due to L. Liu [Uniqueness of meromorphic functions and differential polynomials, Comput. Math. Appl., 56 (2008), 3236-3245.] and P. Sahoo [Uniqueness and weighted value sharing of meromorphic functions, Applied. Math. E-Notes., 11 (2011), 23-32.].
\end{abstract}

\section{Introduction, definitions and results}

In this paper by meromorphic functions we shall always mean meromorphic functions in the complex plane.

Let $f$ and $g$ be two non-constant meromorphic functions and let $a$ be a finite complex number. We say that $f$ and $g$ share a CM, provided that $f-a$ and $g-a$ have same zeros with same multiplicities. Similarly, we say that $f$ and $g$ share $a$ IM, provided that $f-a$ and $g-a$ have same zeros ignoring

2010 Mathematics Subject Classification: Primary 30D35

Key words and phrases: uniqueness, meromorphic function, nonlinear differential polynomials 
multiplicities. In addition we say that $f$ and $g$ share $\infty \mathrm{CM}$, if $1 / \mathrm{f}$ and $1 / \mathrm{g}$ share $0 \mathrm{CM}$, and we say that $f$ and $g$ share $\infty \mathrm{IM}$, if $1 / \mathrm{f}$ and $1 / \mathrm{g}$ share $0 \mathrm{IM}$.

We adopt the standard notations of value distribution theory (see [6]). We denote by $T(r)$ the maximum of $T(r, f)$ and $T(r, g)$. The notation $S(r)$ denotes any quantity satisfying $S(r)=o(T(r))$ as $r \longrightarrow \infty$, outside of a possible exceptional set of finite linear measure.

A meromorphic function $a(z)$ is called a small function with respect to $f$, provided that $T(r, a)=S(r, f)$.

Let $f(z)$ and $g(z)$ be two non-constant meromorphic functions. Let $a(z)$ be a small function with respect to $f(z)$ and $g(z)$. We say that $f(z)$ and $g(z)$ share $a(z)$ CM (counting multiplicities) if $f(z)-a(z)$ and $g(z)-a(z)$ have same zeros with same multiplicities and we say that $f(z), g(z)$ share $a(z)$ IM (ignoring multiplicities) if we do not consider the multiplicities.

Throughout this paper, we need the following definition.

$$
\Theta(a ; f)=1-\limsup _{r \longrightarrow \infty} \frac{\bar{N}(r, a ; f)}{T(r, f)},
$$

where $\mathrm{a}$ is a value in the extended complex plane.

In 1959, W. K. Hayman (see [6], Corollary of Theorem 9) proved the following theorem.

Theorem A Let $\mathrm{f}$ be a transcendental meromorphic function and $\mathrm{n}(\geq 3)$ is an integer. Then $\mathrm{f}^{n} \mathrm{f}^{\prime}=1$ has infinitely many solutions.

Fang and Hua [3], Yang and Hua [16] got a unicity theorem respectively corresponding Theorem A.

Theorem $\mathbf{B}$ Let $\mathrm{f}$ and $\mathrm{g}$ be two non-constant entire (meromorphic) functions, $\mathrm{n} \geq 6(\geq 11)$ be a positive integer. If $\mathrm{f}^{\mathrm{n}} \mathrm{f}^{\prime}$ and $\mathrm{g}^{\mathrm{n}} \mathrm{g}^{\prime}$ share $1 C M$, then either $\mathrm{f}(z)=\mathrm{c}_{1} \mathrm{e}^{\mathrm{cz}}, \mathrm{g}(z)=\mathrm{c}_{2} \mathrm{e}^{-\mathrm{c} z}$, where $\mathrm{c}_{1}, \mathrm{c}_{2}$ and $\mathrm{c}$ are three constants satisfying $\left(\mathrm{c}_{1} \mathrm{c}_{2}\right)^{\mathrm{n}+1} \mathrm{c}^{2}=-1$ or $\mathrm{f} \equiv \mathrm{tg}$ for a constant $\mathrm{t}$ such that $\mathrm{t}^{\mathrm{n}+1}=1$.

Noting that $f^{n}(z) f^{\prime}(z)=\frac{1}{n+1}\left(f^{n+1}(z)\right)^{\prime}$, Fang [4] considered the case of $k$-th derivative and proved the following results.

Theorem $\mathbf{C}$ Let $\mathrm{f}$ and $\mathrm{g}$ be two non-constant entire functions, and let $\mathrm{n}, \mathrm{k}$ be two positive integers with $\mathrm{n}>2 \mathrm{k}+4$. If $\left(\mathrm{f}^{\mathrm{n}}\right)^{(\mathrm{k})}$ and $\left(\mathrm{g}^{\mathrm{n}}\right)^{(\mathrm{k})}$ share $1 C M$, then either $\mathrm{f}(z)=\mathrm{c}_{1} e^{\mathrm{cz}}, \mathrm{g}(z)=\mathrm{c}_{2} e^{-\mathrm{cz} z}$, where $\mathrm{c}_{1}, \mathrm{c}_{2}$ and $\mathrm{c}$ are three constants satisfying $(-1)^{\mathrm{k}}\left(\mathrm{c}_{1} \mathrm{c}_{2}\right)^{\mathrm{n}}(\mathrm{nc})^{2 \mathrm{k}}=1$ or $\mathrm{f} \equiv \mathrm{tg}$ for a constant $\mathrm{t}$ such that $\mathrm{t}^{\mathrm{n}}=1$. 
Theorem $\mathbf{D}$ Let $\mathbf{f}$ and $\mathrm{g}$ be two non-constant entire functions, and let $\mathrm{n}, \mathrm{k}$ be two positive integers with $\mathrm{n}>2 \mathrm{k}+8$. If $\left(\mathrm{f}^{\mathrm{n}}(z)(\mathrm{f}(z)-1)\right)^{(\mathrm{k})}$ and $\left(\mathrm{g}^{\mathrm{n}}(z)(\mathrm{g}(z)-\right.$ 1) $)^{(\mathrm{k})}$ share $1 C M$, then $\mathrm{f}(z) \equiv \mathrm{g}(z)$.

In 2008, X. Y. Zhang and W. C. Lin [21] proved the following result.

Theorem $\mathbf{E}$ Let $\mathbf{f}$ and $\mathrm{g}$ be two non-constant entire functions, and let $\mathrm{n}, \mathrm{m}$ and $\mathrm{k}$ be three positive integers with $\mathrm{n}>2 \mathrm{k}+\mathrm{m}+4$. If $\left[\mathrm{f}^{\mathrm{n}}(\mathrm{f}-1)^{\mathrm{m}}\right]^{(\mathrm{k})}$ and $\left[\mathrm{g}^{\mathrm{n}}(\mathrm{g}-1)^{\mathrm{m}}\right]^{(\mathrm{k})}$ share $1 C M$, then either $\mathrm{f} \equiv \mathrm{g}$ or $\mathrm{f}$ and $\mathrm{g}$ satisfy the algebraic equation $\mathrm{R}(\mathrm{f}, \mathrm{g})=0$, where $\mathrm{R}\left(\omega_{1}, \omega_{2}\right)=\omega_{1}^{\mathrm{n}}\left(\omega_{1}-1\right)^{\mathrm{m}}-\omega_{2}^{\mathrm{n}}(\omega-1)^{\mathrm{m}}$.

In 2001 an idea of gradation of sharing of values was introduced in ([7], [8]) which measures how close a shared value is to being share CM or to being shared IM. This notion is known as weighted sharing and is defined as follows.

Definition $1[7,8]$ Let $\mathrm{k}$ be a nonnegative integer or infinity. For $\mathrm{a} \in \mathbb{C} \cup$ $\{\infty\}$ we denote by $\mathrm{E}_{\mathrm{k}}(\mathrm{a} ; \mathrm{f})$ the set of all $\mathrm{a}$-points of $\mathrm{f}$, where an a-point of multiplicity $\mathrm{m}$ is counted $\mathrm{m}$ times if $\mathrm{m} \leq \mathrm{k}$ and $\mathrm{k}+1$ times if $\mathrm{m}>\mathrm{k}$. If $E_{k}(a ; f)=E_{k}(a ; g)$, we say that $f, g$ share the value a with weight $k$.

The definition implies that if $f, g$ share a value a with weight $k$ then $z_{0}$ is an a-point of $f$ with multiplicity $m(\leq k)$ if and only if it is an a-point of $g$ with multiplicity $m(\leq k)$ and $z_{0}$ is an a-point of $f$ with multiplicity $m(>k)$ if and only if it is an a-point of $g$ with multiplicity $n(>k)$, where $m$ is not necessarily equal to $n$.

We write $f, g$ share $(a, k)$ to mean that $f, g$ share the value $a$ with weight $k$. Clearly if $f, g$ share $(a, k)$, then $f, g$ share $(a, p)$ for any integer $p, 0 \leq p<k$. Also we note that $f, g$ share a value $a$ IM or CM if and only if $f, g$ share $(a, 0)$ or $(a, \infty)$ respectively.

If $a(z)$ is a small function with respect to $f(z)$ and $g(z)$, we define that $f(z)$ and $g(z)$ share $a(z)$ IM or $a(z)$ CM or with weight $l$ according as $f(z)-a(z)$ and $g(z)-a(z)$ share $(0,0)$ or $(0, \infty)$ or $(0, l)$ respectively.

In 2008, L. Liu [12] proved the following.

Theorem $\mathbf{F}$ Let $\mathrm{f}$ and $\mathrm{g}$ be two non-constant entire functions, and let $\mathrm{n}, \mathrm{m}$ and $\mathrm{k}$ be three positive integers such that $\mathrm{n}>5 \mathrm{k}+4 \mathrm{~m}+9$. If $\mathrm{E}_{0}\left(1,\left[\mathrm{f}^{\mathrm{n}}(\mathrm{f}-\right.\right.$ $\left.\left.1)^{\mathrm{m}}\right]^{(\mathrm{k})}\right)=\mathrm{E}_{0}\left(1,\left[\mathrm{~g}^{\mathrm{n}}(\mathrm{g}-1)^{\mathrm{m}}\right]^{(\mathrm{k})}\right)$ then either $\mathrm{f} \equiv \mathrm{g}$ or $\mathrm{f}$ and $\mathrm{g}$ satisfy the algebraic equation $\mathrm{R}(\mathrm{f}, \mathrm{g})=0$, where $\mathrm{R}\left(\omega_{1}, \omega_{2}\right)=\omega_{1}^{\mathrm{n}}\left(\omega_{1}-1\right)^{\mathrm{m}}-\omega_{2}^{\mathrm{n}}\left(\omega_{2}-1\right)^{\mathrm{m}}$.

Recently P. Sahoo [14] proved the following result.

Theorem $\mathbf{G}$ Let $\mathrm{f}$ and $\mathrm{g}$ be two transcendental meromorphic functions and $\mathrm{n}(\geq 1), \mathrm{k}(\geq 1), \mathrm{m}(\geq 0)$ and $\mathrm{l}(\geq 0)$ be four integers. Let $\left[\mathrm{f}^{\mathrm{n}}(\mathrm{f}-1)^{\mathrm{m}}\right]^{(\mathrm{k})}$ and $\left[\mathrm{g}^{\mathrm{n}}(\mathrm{g}-1)^{\mathrm{m}}\right]^{(\mathrm{k})}$ share $(\mathrm{b}, \mathrm{l})$ for a nonzero constant $\mathrm{b}$. Then 
(1) when $\mathrm{m}=0$, if $\mathrm{f}(z) \neq \infty, \mathrm{g}(z) \neq \infty$ and $\mathrm{l} \geq 2$, $\mathrm{n}>3 \mathrm{k}+8$ or $\mathrm{l}=1$, $\mathrm{n}>5 \mathrm{k}+10$ or $\mathrm{l}=0, \mathrm{n}>9 \mathrm{k}+14$, then either $\mathrm{f} \equiv \mathrm{tg}$, where $\mathrm{t}$ is a constant satisfying $\mathrm{t}^{\mathrm{n}}=1$, or $\mathrm{f}(z)=\mathrm{c}_{1} \mathrm{e}^{\mathrm{cz}}, \mathrm{g}(z)=\mathrm{c}_{2} e^{-\mathrm{cz} z}$, where $\mathrm{c}_{1}, \mathrm{c}_{2}$ and $\mathrm{c}$ are three constants satisfying $(-1)^{\mathrm{k}}\left(\mathrm{c}_{1} \mathrm{c}_{2}\right)^{\mathrm{n}}(\mathrm{nc})^{2 \mathrm{k}}=\mathrm{b}^{2}$,

(2) when $\mathrm{m}=1$ and $\Theta(\infty ; f)>\frac{2}{n}$ then either $\left[\mathrm{f}^{\mathrm{n}}(\mathrm{f}-1)\right]^{(\mathrm{k})}\left[\mathrm{g}^{\mathrm{n}}(\mathrm{g}-1)\right]^{(\mathrm{k})} \equiv \mathrm{b}^{2}$, except for $\mathrm{k}=1$ or $\mathrm{f} \equiv \mathrm{g}$, provided one of $\mathrm{l} \geq 2, \mathrm{n}>3 \mathrm{k}+11$ or $\mathrm{l}=1$, $\mathrm{n}>5 \mathrm{k}+14$ orl $=0, \mathrm{n}>9 \mathrm{k}+20$ holds; and

(3) when $m \geq 2$, and $l \geq 2, n>3 k+m+10$ or $l=1, n>5 k+2 m+12$ or $\mathrm{l}=0, \mathrm{n}>9 \mathrm{k}+4 \mathrm{~m}+16$, then either $\left[\mathrm{f}^{\mathrm{n}}(\mathrm{f}-1)^{\mathrm{m}}\right]^{(\mathrm{k})}\left[\mathrm{g}^{\mathrm{n}}(\mathrm{g}-1)^{\mathrm{m}}\right]^{(\mathrm{k})} \equiv \mathrm{b}^{2}$ except for $\mathrm{k}=1$ or $\mathrm{f} \equiv \mathrm{g}$ or $\mathrm{f}$ and $\mathrm{g}$ satisfying the algebraic equation $\mathrm{R}(\mathrm{f}, \mathrm{g})=0$, where

$$
R\left(\omega_{1}, \omega_{2}\right)=\omega_{1}^{\mathrm{n}}\left(\omega_{1}-1\right)^{\mathrm{m}}-\omega_{2}^{\mathrm{n}}\left(\omega_{2}-1\right)^{\mathrm{m}} .
$$

It is quite natural to ask the following questions.

Question 1: Can lower bound of $n$ be further reduced in Theorems $F$, G?

Question 2: Can one remove the condition $f \neq \infty, g \neq \infty$ when $m=0$ in Theorem G?

In this paper, taking the possible answer of the above questions into background we obtain the following results which improve and generalize Theorems F, G.

Theorem 1 Let $\mathrm{f}$ and $\mathrm{g}$ be two transcendental meromorphic functions and let $\mathrm{p}(\mathrm{z})$ be a nonzero polynomial with $\operatorname{deg}(\mathrm{p})=\mathrm{l}$. Suppose $\left[\mathrm{f}^{\mathrm{n}}(\mathrm{f}-1)^{\mathrm{m}}\right]^{(\mathrm{k})}-\mathrm{p}$ and $\left[g^{n}(g-1)^{m}\right]^{(k)}-p$ share $\left(0, k_{1}\right)$, where $n(\geq 1), k(\geq 1), m(\geq 0)$ are three integers. Now when one of the following conditions holds:

(i) $k_{1} \geq 2$ and $n>3 k+m+8\left(=s_{2}\right)$;

(ii) $\mathrm{k}_{1}=1$ and $\mathrm{n}>4 \mathrm{k}+\frac{3 \mathrm{~m}}{2}+9\left(=\mathrm{s}_{1}\right)$;

(iii) $\mathrm{k}_{1}=0$ and $\mathrm{n}>9 \mathrm{k}+4 \mathrm{~m}+14\left(=\mathrm{s}_{0}\right)$;

then the following conclusions occur

(1) when $\mathrm{m}=0$, then either $\mathrm{f} \equiv \mathrm{tg}$, where $\mathrm{t}$ is a constant satisfying $\mathrm{t}^{\mathrm{n}}=1$, or if $\mathrm{p}(z)$ is not a constant and $\mathrm{n}>\max \left\{s_{i}, 2 k+2 l-1\right\}, i=0,1,2$, then $\mathrm{f}(z)=\mathrm{c}_{1} e^{\mathrm{cQ}(z)}, \mathrm{g}(z)=\mathrm{c}_{2} e^{-\mathrm{cQ}(z)}$, where $\mathrm{Q}(z)=\int_{0}^{z} \mathrm{p}(z) \mathrm{d} z, \mathrm{c}_{1}, \mathrm{c}_{2}$ and $\mathrm{c}$ are constants such that $(\mathrm{nc})^{2}\left(\mathrm{c}_{1} \mathrm{c}_{2}\right)^{\mathrm{n}}=-1$, if $\mathrm{p}(z)$ is a nonzero constant $\mathrm{b}$, then $\mathrm{f}(z)=\mathrm{c}_{3} e^{\mathrm{d} z}, \mathrm{~g}(z)=\mathrm{c}_{4} e^{-\mathrm{d} z}$, where $\mathrm{c}_{3}, \mathrm{c}_{4}$ and $\mathrm{d}$ are constants such that $(-1)^{\mathrm{k}}\left(\mathrm{c}_{3} \mathrm{c}_{4}\right)^{\mathrm{n}}(\mathrm{nd})^{2 \mathrm{k}}=\mathrm{b}^{2}$; 
(2) when $\mathrm{m}=1$ and $\Theta(\infty ; f)+\Theta(\infty ; g)>\frac{4}{n}$, then either $\left[\mathrm{f}^{\mathrm{n}}(\mathrm{f}-1)\right]^{(\mathrm{k})}\left[\mathrm{g}^{\mathrm{n}}(\mathrm{g}-\right.$ $1)]^{(\mathrm{k})} \equiv \mathrm{p}^{2}$, except for $\mathrm{k}=1$ or $\mathrm{f} \equiv \mathrm{g}$

(3) when $m \geq 2$, then either $\left[f^{\mathfrak{n}}(f-1)^{m}\right]^{(k)}\left[g^{n}(g-1)^{m}\right]^{(k)} \equiv p^{2}$ except for $\mathrm{k}=1$ or $\mathrm{f} \equiv \mathrm{g}$ or $\mathrm{f}$ and $\mathrm{g}$ satisfying the algebraic equation $\mathrm{R}(\mathrm{f}, \mathrm{g})=0$, where

$$
R\left(\omega_{1}, \omega_{2}\right)=\omega_{1}^{n}\left(\omega_{1}-1\right)^{m}-\omega_{2}^{n}\left(\omega_{2}-1\right)^{m} .
$$

In addition, when $\mathrm{f}$ and $\mathrm{g}$ share $(\infty, 0)$, then the possibility $\left[\mathrm{f}^{\mathrm{n}}(\mathrm{f}-1)^{\mathrm{m}}\right]^{(\mathrm{k})}\left[\mathrm{g}^{\mathrm{n}}\right.$ $\left.(\mathrm{g}-1)^{\mathrm{m}}\right]^{(\mathrm{k})} \equiv \mathrm{p}^{2}$ does not occur for $\mathrm{m} \geq 1$.

Remark 1 When $\mathrm{f}$ and $\mathrm{g}$ share $\infty$ IM then the conditions (i), (ii) and (iii) of Theorem 1 will be replaced by respectively $l \geq 2$ and $n>3 k+m+7, l=1$ and $\mathrm{n}>4 \mathrm{k}+\frac{3 \mathrm{~m}}{2}+8$ and $\mathrm{l}=0$ and $\mathrm{n}>9 \mathrm{k}+4 \mathrm{~m}+13$.

Theorem 2 Let $\mathrm{f}$ and $\mathrm{g}$ be two transcendental entire functions and let $\mathrm{p}(z)$ be a nonzero polynomial with $\operatorname{deg}(\mathrm{p})=\mathrm{l}$. Suppose $\left[\mathrm{f}^{\mathrm{n}}(\mathrm{f}-1)^{\mathrm{m}}\right]^{(\mathrm{k})}-\mathrm{p}$ and $\left[g^{n}(g-1)^{m}\right]^{(k)}-p$ share $\left(0, k_{1}\right)$, where $n(\geq 1), k(\geq 1), m(\geq 0)$ are three integers. Now when one of the following conditions holds:

(i) $k_{1} \geq 2$ and $n>2 k+m+4\left(=s_{2}\right)$;

(ii) $\mathrm{k}_{1}=1$ and $\mathrm{n}>\frac{5 \mathrm{k}+3 \mathrm{~m}+9}{2}\left(=\mathrm{s}_{1}\right)$;

(iii) $\mathrm{k}_{1}=0$ and $\mathrm{n}>5 \mathrm{k}+4 \mathrm{~m}+7\left(=\mathrm{s}_{0}\right)$;

then the following conclusions occur

(1) when $\mathrm{m}=0$, then either $\mathrm{f} \equiv \mathrm{tg}$, where $\mathrm{t}$ is a constant satisfying $\mathrm{t}^{\mathrm{n}}=1$, or if $\mathrm{p}(z)$ is not a constant and $\mathrm{n}>\max \left\{\mathrm{s}_{i}, \mathrm{k}+2 \mathrm{l}\right\}, \mathrm{i}=0,1,2$, then $\mathrm{f}(z)=\mathrm{c}_{1} \mathrm{e}^{\mathrm{cQ}(z)}, \mathrm{g}(z)=\mathrm{c}_{2} \mathrm{e}^{-\mathrm{cQ}(z)}$, where $\mathrm{Q}(z)=\int_{0}^{z} \mathrm{p}(z) \mathrm{d} z, \mathrm{c}_{1}, \mathrm{c}_{2}$ and $\mathrm{c}$ are constants such that $(\mathrm{nc})^{2}\left(\mathrm{c}_{1} \mathrm{c}_{2}\right)^{\mathrm{n}}=-1$,

if $\mathrm{p}(z)$ is a nonzero constant $\mathrm{b}$, then $\mathrm{f}(z)=\mathrm{c}_{3} \mathrm{e}^{\mathrm{d} z}, \mathrm{~g}(z)=\mathrm{c}_{4} \mathrm{e}^{-\mathrm{d} z}$, where $\mathrm{c}_{3}, \mathrm{c}_{4}$ and $\mathrm{d}$ are constants such that $(-1)^{\mathrm{k}}\left(\mathrm{c}_{3} \mathrm{c}_{4}\right)^{\mathrm{n}}(\mathrm{nd})^{2 \mathrm{k}}=\mathrm{b}^{2}$;

(2) when $\mathrm{m}=1$ then $\mathrm{f} \equiv \mathrm{g}$;

(3) when $\mathrm{m} \geq 2$, then either $\mathrm{f} \equiv \mathrm{g}$ or $\mathrm{f}$ and $\mathrm{g}$ satisfying the algebraic equation $\mathrm{R}(\mathrm{f}, \mathrm{g})=0$, where

$$
R\left(\omega_{1}, \omega_{2}\right)=\omega_{1}^{n}\left(\omega_{1}-1\right)^{m}-\omega_{2}^{n}\left(\omega_{2}-1\right)^{m} .
$$

We now explain some definitions and notations which are used in the paper. 
Definition 2 [10] Let $\mathrm{p}$ be a positive integer and $\mathrm{a} \in \mathbb{C} \cup\{\infty\}$.

(i) $N(r, a ; f \mid \geq p)(\bar{N}(r, a ; f \mid \geq p))$ denotes the counting function (reduced counting function) of those a-points of $\mathrm{f}$ whose multiplicities are not less than $\mathrm{p}$.

(ii) $\mathrm{N}(r, a ; f \mid \leq p)(\bar{N}(r, a ; f \mid \leq p))$ denotes the counting function (reduced counting function) of those a-points of $\mathrm{f}$ whose multiplicities are not greater than $\mathrm{p}$.

Definition $3\{11$, cf.[18] $\}$ For $\mathrm{a} \in \mathbb{C} \cup\{\infty\}$ and a positive integer $\mathrm{p}$ we denote by $\mathrm{N}_{p}(r, a ; f)$ the sum $\bar{N}(r, a ; f)+\bar{N}(r, a ; f \mid \geq 2)+\ldots \bar{N}(r, a ; f \mid \geq p)$. Clearly $\mathrm{N}_{1}(\mathrm{r}, \mathrm{a} ; f)=\overline{\mathrm{N}}(\mathrm{r}, \mathrm{a} ; \mathrm{f})$.

Definition 4 Let $\mathrm{a}, \mathrm{b} \in \mathbb{C} \cup\{\infty\}$. Let $\mathrm{p}$ be a positive integer. We denote by $\overline{\mathrm{N}}(r, a ; f|\geq p| g=b)(\bar{N}(r, a ; f|\geq p| g \neq b))$ the reduced counting function of those a-points of $\mathrm{f}$ with multiplicities $\geq \mathrm{p}$, which are the $\mathrm{b}$-points (not the $\mathrm{b}$-points) of $\mathrm{g}$.

Definition 5 \{cf.[1], 2$\}$ Let $\mathbf{f}$ and $\mathrm{g}$ be two non-constant meromorphic functions such that $\mathrm{f}$ and $\mathrm{g}$ share the value 1 IM. Let $z_{0}$ be a 1-point of $\mathrm{f}$ with multiplicity $\mathrm{p}$, a 1-point of $\mathrm{g}$ with multiplicity $\mathrm{q}$. We denote by $\overline{\mathrm{N}}_{\mathrm{L}}(\mathrm{r}, 1 ; \mathrm{f})$ the counting function of those 1 -points of $f$ and $g$ where $p>q$, by $\mathrm{N}_{\mathrm{E}}^{1)}(\mathrm{r}, 1 ; \mathrm{f})$ the counting function of those 1-points of $\mathrm{f}$ and $\mathrm{g}$ where $\mathrm{p}=\mathrm{q}=1$ and by $\overline{\mathrm{N}}_{\mathrm{E}}^{(2}(\mathrm{r}, 1 ; \mathrm{f})$ the counting function of those 1 -points of $\mathrm{f}$ and $\mathrm{g}$ where $\mathrm{p}=\mathrm{q} \geq 2$, each point in these counting functions is counted only once. In the same way we can define $\overline{\mathrm{N}}_{\mathrm{L}}(\mathrm{r}, 1 ; \mathrm{g}), \mathrm{N}_{\mathrm{E}}^{1)}(\mathrm{r}, 1 ; \mathrm{g}), \overline{\mathrm{N}}_{\mathrm{E}}^{(2}(\mathrm{r}, 1 ; \mathrm{g})$.

Definition 6 \{cf.[1], 2$\}$ Let $\mathrm{k}$ be a positive integer. Let $\mathrm{f}$ and $\mathrm{g}$ be two nonconstant meromorphic functions such that $\mathrm{f}$ and $\mathrm{g}$ share the value $1 \mathrm{IM}$. Let $z_{0}$ be a 1-point of $\mathrm{f}$ with multiplicity $\mathrm{p}$, a 1-point of $\mathrm{g}$ with multiplicity $\mathrm{q}$. We denote by $\overline{\mathrm{N}}_{\mathrm{f}>\mathrm{k}}(\mathrm{r}, 1 ; \mathrm{g})$ the reduced counting function of those 1-points of $\mathrm{f}$ and $\mathrm{g}$ such that $\mathrm{p}>\mathrm{q}=\mathrm{k} . \overline{\mathrm{N}}_{\mathrm{g}>\mathrm{k}}(\mathrm{r}, 1 ; \mathrm{f})$ is defined analogously.

Definition 7 [7, 8] Let $\mathrm{f}, \mathrm{g}$ share a value a IM. We denote by $\overline{\mathrm{N}}_{*}(\mathrm{r}, \mathrm{a} ; \mathrm{f}, \mathrm{g})$ the reduced counting function of those a-points of $\mathrm{f}$ whose multiplicities differ from the multiplicities of the corresponding a-points of $\mathrm{g}$.

Clearly $\overline{\mathrm{N}}_{*}(\mathrm{r}, \mathrm{a} ; \mathrm{f}, \mathrm{g}) \equiv \overline{\mathrm{N}}_{*}(\mathrm{r}, \mathrm{a} ; \mathrm{g}, \mathrm{f})$ and $\overline{\mathrm{N}}_{*}(\mathrm{r}, \mathrm{a} ; \mathrm{f}, \mathrm{g})=\overline{\mathrm{N}}_{\mathrm{L}}(\mathrm{r}, \mathrm{a} ; \mathrm{f})+\overline{\mathrm{N}}_{\mathrm{L}}(\mathrm{r}, \mathrm{a} ; \mathrm{g})$. 


\section{Lemmas}

Let $F$ and $G$ be two non-constant meromorphic functions defined in $\mathbb{C}$. We denote by $\mathrm{H}$ the function as follows:

$$
\mathrm{H}=\left(\frac{\mathrm{F}^{\prime \prime}}{\mathrm{F}^{\prime}}-\frac{2 \mathrm{~F}^{\prime}}{\mathrm{F}-1}\right)-\left(\frac{\mathrm{G}^{\prime \prime}}{\mathrm{G}^{\prime}}-\frac{2 \mathrm{G}^{\prime}}{\mathrm{G}-1}\right) .
$$

Lemma 1 [15] Let $\mathrm{f}$ be a non-constant meromorphic function and let $\mathrm{a}_{\mathrm{n}}(z)(\not \equiv$ $0), a_{n-1}(z), \ldots, a_{0}(z)$ be meromorphic functions such that $\mathrm{T}\left(\mathrm{r}, \mathrm{a}_{\mathrm{i}}(z)\right)=\mathrm{S}(\mathrm{r}, \mathrm{f})$ for $\mathrm{i}=0,1,2, \ldots, \mathrm{n}$. Then

$$
T\left(r, a_{n} f^{n}+a_{n-1} f^{n-1}+\ldots+a_{1} f+a_{0}\right)=n T(r, f)+S(r, f) .
$$

Lemma 2 [20] Let $\mathrm{f}$ be a non-constant meromorphic function, and $\mathrm{p}, \mathrm{k}$ be positive integers. Then

$$
\begin{gathered}
N_{p}\left(r, 0 ; f^{(k)}\right) \leq T\left(r, f^{(k)}\right)-T(r, f)+N_{p+k}(r, 0 ; f)+S(r, f), \\
N_{p}\left(r, 0 ; f^{(k)}\right) \leq k \bar{N}(r, \infty ; f)+N_{p+k}(r, 0 ; f)+S(r, f) .
\end{gathered}
$$

Lemma 3 [9] If $\mathrm{N}\left(\mathrm{r}, 0 ; \mathrm{f}^{(\mathrm{k})} \mid \mathrm{f} \neq 0\right)$ denotes the counting function of those zeros of $\mathrm{f}^{(\mathrm{k})}$ which are not the zeros of $\mathrm{f}$, where a zero of $\mathrm{f}^{(\mathrm{k})}$ is counted according to its multiplicity, then

$$
N\left(r, 0 ; f^{(k)} \mid f \neq 0\right) \leq k \bar{N}(r, \infty ; f)+N(r, 0 ; f \mid<k)+k \bar{N}(r, 0 ; f \mid \geq k)+S(r, f) .
$$

Lemma 4 [11] Let $\mathrm{f}_{1}$ and $\mathrm{f}_{2}$ be two non-constant meromorphic functions satisfying $\overline{\mathrm{N}}\left(\mathrm{r}, 0 ; \mathrm{f}_{\mathrm{i}}\right)+\overline{\mathrm{N}}\left(\mathrm{r}, \infty ; \mathrm{f}_{\mathrm{i}}\right)=\mathrm{S}\left(\mathrm{r} ; \mathrm{f}_{1}, \mathrm{f}_{2}\right)$ for $\mathrm{i}=1$, 2. If $\mathrm{f}_{1}^{\mathrm{s}} \mathrm{f}_{2}^{\mathrm{t}}-1$ is not identically zero for arbitrary integers $\mathrm{s}$ and $\mathrm{t}(|\boldsymbol{s}|+|\mathrm{t}|>0)$, then for any positive $\varepsilon$, we have

$$
N_{0}\left(r, 1 ; f_{1}, f_{2}\right) \leq \varepsilon T(r)+S\left(r ; f_{1}, f_{2}\right),
$$

where $\mathrm{N}_{0}\left(\mathrm{r}, 1 ; \mathrm{f}_{1}, \mathrm{f}_{2}\right)$ denotes the deduced counting function related to the common 1-points of $\mathrm{f}_{1}$ and $\mathrm{f}_{2}$ and $\mathrm{T}(\mathrm{r})=\mathrm{T}\left(\mathrm{r}, \mathrm{f}_{1}\right)+\mathrm{T}\left(\mathrm{r}, \mathrm{f}_{2}\right), \mathrm{S}\left(\mathrm{r} ; \mathrm{f}_{1}, \mathrm{f}_{2}\right)=\mathrm{o}(\mathrm{T}(\mathrm{r}))$ as $\mathrm{r} \longrightarrow \infty$ possibly outside a set of finite linear measure.

Lemma 5 [6] Suppose that $\mathrm{f}$ is a non-constant meromorphic function, $\mathrm{k} \geq 2$ is an integer. If

$$
N(r, \infty, f)+N(r, 0 ; f)+N\left(r, 0 ; f^{(k)}\right)=S\left(r, \frac{f^{\prime}}{f}\right),
$$

then $\mathrm{f}(z)=\mathrm{e}^{\mathrm{a} z+\mathrm{b}}$, where $\mathrm{a} \neq 0, \mathrm{~b}$ are constants. 
Lemma 6 [5] Let $\mathrm{f}(\mathrm{z})$ be a non-constant entire function and let $\mathrm{k} \geq 2$ be a positive integer. If $\mathrm{f}(z) \mathrm{f}^{(\mathrm{k})}(z) \neq 0$, then $\mathrm{f}(z)=e^{\mathrm{a} z+\mathrm{b}}$, where $\mathrm{a} \neq 0, \mathrm{~b}$ are constant.

Lemma 7 [19] Let $\mathrm{f}$ be a non-constant meromorphic function, and let $\mathrm{k}$ be a positive integer. Suppose that $\mathrm{f}^{(\mathrm{k})} \not \equiv 0$, then

$$
N\left(r, 0 ; f^{(k)}\right) \leq N(r, 0 ; f)+k \bar{N}(r, \infty ; f)+S(r, f) .
$$

Lemma 8 Let $\mathrm{f}$ and $\mathrm{g}$ be two non-constant meromorphic functions. Let $\mathrm{n}(\geq$ $1), k(\geq 1)$ and $m(\geq 0)$ be three integers such that $n>3 k+m+1$. If $\left[f^{n}(f-1)^{m}\right]^{(k)} \equiv\left[g^{n}(g-1)^{m}\right]^{(k)}$, then $f^{n}(f-1)^{m} \equiv g^{n}(g-1)^{m}$.

Proof. We have $\left[f^{\mathfrak{n}}(f-1)^{m}\right]^{(k)} \equiv\left[g^{\mathfrak{n}}(g-1)^{m}\right]^{(k)}$. Integrating we get

$$
\left[f^{\mathfrak{n}}(f-1)^{m}\right]^{(k-1)} \equiv\left[g^{\mathfrak{n}}(g-1)^{m}\right]^{(k-1)}+c_{k-1} .
$$

If possible suppose $c_{k-1} \neq 0$. Now in view of Lemma 2 for $p=1$ and using second fundamental theorem we get

$$
\begin{aligned}
& (n+m) T(r, f) \\
\leq & T\left(r,\left[f^{n}(f-1)^{m}\right]^{(k-1)}\right)-\bar{N}\left(r, 0 ;\left[f^{n}(f-1)^{m}\right]^{(k-1)}\right)+N_{k}\left(r, 0 ; f^{n}(f-1)^{m}\right) \\
& +S(r, f) \\
\leq & \bar{N}\left(r, 0 ;\left[f^{n}(f-1)^{m}\right]^{(k-1)}\right)+\bar{N}(r, \infty ; f)+\bar{N}\left(r, c_{k-1} ;\left[f^{n}(f-1)^{m}\right]^{(k-1)}\right) \\
& -\bar{N}\left(r, 0 ;\left[f^{n}(f-1)^{m}\right]^{(k-1)}\right)+N_{k}\left(r, 0 ; f^{n}(f-1)^{m}\right)+S(r, f) \\
\leq & \bar{N}(r, \infty ; f)+\bar{N}\left(r, 0 ;\left[g^{n}(g-1)^{m}\right]^{(k-1)}\right)+k \bar{N}(r, 0 ; f)+N\left(r, 0 ;(f-1)^{m}\right) \\
& +S(r, f) \\
\leq & (k+1+m) T(r, f)+(k-1) \bar{N}(r, \infty ; g)+N_{k}\left(r, 0 ; g^{n}(g-1)^{m}\right)+S(r, f) \\
\leq & (k+1+m) T(r, f)+k \bar{N}(r, \infty ; g)+k \bar{N}(r, 0 ; g)+N\left(r, 0 ;(g-1)^{m}\right) \\
& +S(r, f) \\
\leq & (k+1+m) T(r, f)+(2 k+m) T(r, g)+S(r, f)+S(r, g) \\
\leq & (3 k+2 m+1) T(r)+S(r) .
\end{aligned}
$$

Similarly we get

$$
(n+m) T(r, g) \leq(3 k+2 m+1) T(r)+S(r) .
$$

Combining these we get

$$
(n-m-3 k-1) T(r) \leq S(r),
$$


which is a contradiction since $n>3 k+m+1$. Therefore $c_{k-1}=0$ and so

$$
\left[f^{n}(f-1)^{m}\right]^{(k-1)} \equiv\left[g^{n}(g-1)^{m}\right]^{(k-1)} \text {. }
$$

Proceeding in this way we obtain

$$
\left[f^{n}(f-1)^{m}\right]^{\prime} \equiv\left[g^{n}(g-1)^{m}\right]^{\prime} .
$$

Integrating we get

$$
f^{n}(f-1)^{m} \equiv g^{n}(g-1)^{m}+c_{0} .
$$

If possible suppose $c_{0} \neq 0$. Now using second fundamental theorem we get

$$
\begin{aligned}
& (n+m) T(r, f) \\
\leq & \bar{N}\left(r, 0 ; f^{n}(f-1)^{m}\right)+\bar{N}\left(r, \infty ; f^{n}(f-1)^{m}\right)+\bar{N}\left(r, c_{0} ; f^{n}(f-1)^{m}\right)+S(r, f) \\
\leq & \bar{N}(r, 0 ; f)+m T(r, f)+\bar{N}(r, \infty ; f)+\bar{N}\left(r, 0 ; g^{n}(g-1)^{m}\right)+S(r, f) \\
\leq & (m+1) T(r, f)+\bar{N}(r, \infty ; f)+\bar{N}(r, 0 ; g)+m T(r, g)+S(r, f) \\
\leq & (3+2 m) T(r)+S(r) .
\end{aligned}
$$

Similarly we get

$$
(n+m) T(r, g) \leq(3+2 m) T(r)+S(r) .
$$

Combining these we get

$$
(n-3-m) T(r) \leq S(r),
$$

which is a contradiction since $n>4+m$. Therefore $c_{0}=0$ and so

$$
f^{n}(f-1)^{m} \equiv g^{n}(g-1)^{m} .
$$

This proves the Lemma.

Lemma 9 Let $\mathrm{f}, \mathrm{g}$ be two transcendental meromorphic functions, let $\mathrm{n}(\geq 1)$, $\mathrm{m}(\geq 0)$ and $\mathrm{k}(\geq 1)$ be three integers with $\mathrm{n}>\mathrm{k}+2$. If $\left[\mathrm{f}^{\mathrm{n}}(\mathrm{f}-1)^{\mathrm{m}}\right]^{(\mathrm{k})}-\mathrm{p}$ and $\left[\mathrm{g}^{\mathrm{n}}(\mathrm{g}-1)^{\mathrm{m}}\right]^{(\mathrm{k})}-\mathrm{p}$ share $(0,0)$, where $\mathrm{p}(\mathrm{z})$ is a non zero polynomial, then $\mathrm{T}(\mathrm{r}, \mathrm{f})=\mathrm{O}(\mathrm{T}(\mathrm{r}, \mathrm{g}))$ and $\mathrm{T}(\mathrm{r}, \mathrm{g})=\mathrm{O}(\mathrm{T}(\mathrm{r}, \mathrm{f}))$. 
Proof. In view of Lemmas 1, 2 for $p=1$ and using second fundamental theorem for small function (see [17]) we get

$$
\begin{aligned}
& (n+m) T(r, f)=T\left(r, f^{n}(f-1)^{m}\right)+O(1) \\
\leq & T\left(r,\left[f^{n}(f-1)^{m}\right]^{(k)}\right)-\bar{N}\left(r, 0 ;\left[f^{n}(f-1)^{m}\right]^{(k)}\right)+N_{k+1}\left(r, 0 ; f^{n}(f-1)^{m}\right) \\
& +S(r, f) \\
\leq & \bar{N}\left(r, 0 ;\left[f^{n}(f-1)^{m}\right]^{(k)}\right)+\bar{N}(r, \infty ; f)+\bar{N}\left(r, p ;\left[f^{n}(f-1)^{m}\right]^{(k)}\right) \\
& -\bar{N}\left(r, 0 ;\left[f^{n}(f-1)^{m}\right]^{(k)}\right)+N_{k+1}\left(r, 0 ; f^{n}(f-1)^{m}\right)+(\varepsilon+o(1)) T(r, f) \\
\leq & \bar{N}(r, \infty ; f)+\bar{N}\left(r, p ;\left[f^{n}(f-1)^{m}\right]^{(k)}\right)+(k+1) \bar{N}(r, 0 ; f)+N\left(r, 0 ;(f-1)^{m}\right) \\
& +(\varepsilon+o(1)) T(r, f) \\
\leq \quad & (k+2+m) T(r, f)+\bar{N}\left(r, p ;\left[g^{n}(g-1)^{m}\right]^{(k)}\right)+(\varepsilon+o(1)) T(r, f) \\
\leq & (k+2+m) T(r, f)+(k+1)(n+m) T(r, g)+(\varepsilon+o(1)) T(r, f),
\end{aligned}
$$

i.e.,

$$
(n-k-2) T(r, f) \leq(k+1)(n+m) T(r, g)+(\varepsilon+o(1)) T(r, f),
$$

for all $\varepsilon>0$. Take $\varepsilon<1$. Since $n>k+2$, we have $T(r, f)=O(T(r, g))$. Similarly we have $\mathrm{T}(\mathrm{r}, \mathrm{g})=\mathrm{O}(\mathrm{T}(\mathrm{r}, \mathrm{f}))$. This completes the proof.

Lemma 10 Let $\mathrm{f}, \mathrm{g}$ be two transcendental meromorphic functions and let $\mathrm{F}=\frac{\left[\mathrm{f}^{\mathrm{n}}(\mathrm{f}-1)^{\mathrm{m}}\right]^{(\mathrm{k})}}{\mathrm{p}}, \mathrm{G}=\frac{\left[\mathrm{g}^{\mathrm{n}}(\mathrm{g}-1)^{\mathrm{m}}\right]^{(\mathrm{k})}}{\mathrm{p}}$, where $\mathrm{p}(\mathrm{z})$ is a non zero polynomial and $\mathrm{n}(\geq 1), \mathrm{k}(\geq 1)$ and $\mathrm{m}(\geq 0)$ are three integers such that $\mathrm{n}>3 \mathrm{k}+\mathrm{m}+3$. If $\mathrm{H} \equiv 0$, then $\left[\mathrm{f}^{\mathrm{n}}(\mathrm{f}-1)^{\mathrm{m}}\right]^{(\mathrm{k})}-\mathrm{p}$ and $\left[\mathrm{g}^{\mathrm{n}}(\mathrm{g}-1)^{\mathrm{m}}\right]^{(\mathrm{k})}-\mathrm{p}$ share $(0, \infty)$ as well as one of the following conclusions occur

(i) $\left[f^{n}(f-1)^{m}\right]^{(k)}\left[g^{n}(g-1)^{m}\right]^{(k)} \equiv p^{2}$;

(ii) $f^{n}(f-1)^{m} \equiv g^{n}(g-1)^{m}$.

Proof. Let $\mathrm{P}(w)=(w-1)^{\mathrm{m}}$. Then $\mathrm{F}=\frac{\left[\mathrm{f}^{\mathrm{n}} \mathrm{P}(\mathrm{f})\right]^{(\mathrm{k})}}{\mathrm{p}}$ and $\mathrm{G}=\frac{\left[g^{\mathrm{n}} \mathrm{P}(\mathrm{g})\right]^{(\mathrm{k})}}{\mathrm{p}}$.

Since $\mathrm{H} \equiv 0$, by integration we get

$$
\frac{1}{F-1} \equiv \frac{B G+A-B}{G-1},
$$

where $A, B$ are constants and $A \neq 0$. From (4) it is clear that $F$ and $G$ share $(1, \infty)$. We now consider following cases.

Case 1. Let $B \neq 0$ and $A \neq B$.

If $\mathrm{B}=-1$, then from (4) we have

$$
\mathrm{F} \equiv \frac{-A}{\mathrm{G}-\mathrm{A}-1} .
$$


Therefore

$$
\bar{N}(r, A+1 ; G)=\bar{N}(r, \infty ; F)=\bar{N}(r, \infty ; f)+\bar{N}(r, 0 ; p) .
$$

So in view of Lemmas 1, 2 and the second fundamental theorem we get

$$
\begin{aligned}
& (n+m) T(r, g) \\
\leq & T(r, G)+N_{k+1}\left(r, 0 ; g^{n} P(g)\right)-\bar{N}(r, 0 ; G)+S(r, g) \\
\leq & \bar{N}(r, \infty ; G)+\bar{N}(r, 0 ; G)+\bar{N}(r, A+1 ; G)+N_{k+1}\left(r, 0 ; g^{n} P(g)\right) \\
& -\bar{N}(r, 0 ; G)+S(r, g) \\
\leq & \bar{N}(r, \infty ; g)+N_{k+1}\left(r, 0 ; g^{n} P(g)\right)+\bar{N}(r, \infty ; f)+S(r, g) \\
\leq & \bar{N}(r, \infty ; f)+\bar{N}(r, \infty ; g)+N_{k+1}\left(r, 0 ; g^{n}\right)+N_{k+1}(r, 0 ; P(g))+S(r, g) \\
\leq & \bar{N}(r, \infty ; f)+\bar{N}(r, \infty ; g)+(k+1) \bar{N}(r, 0 ; g)+T(r, P(g))+S(r, g) \\
\leq & T(r, f)+(k+2+m) T(r, g)+S(r, f)+S(r, g) .
\end{aligned}
$$

Without loss of generality, we suppose that there exists a set I with infinite measure such that $T(r, f) \leq T(r, g)$ for $r \in I$.

So for $r \in I$ we have

$$
(n-k-3) T(r, g) \leq S(r, g)
$$

which is a contradiction since $\mathrm{n}>\mathrm{k}+3$.

If $B \neq-1$, from (4) we obtain that

$$
F-\left(1+\frac{1}{B}\right) \equiv \frac{-A}{B^{2}\left[G+\frac{A-B}{B}\right]} .
$$

So

$$
\bar{N}\left(r, \frac{(B-A)}{B} ; G\right)=\bar{N}(r, \infty ; F)=\bar{N}(r, \infty ; f)+\bar{N}(r, 0 ; p) .
$$

Using Lemmas 1, 2 and the same argument as used in the case when $B=-1$ we can get a contradiction.

Case 2. Let $B \neq 0$ and $A=B$.

If $B=-1$, then from (4) we have

$$
\mathrm{FG} \equiv 1
$$

i.e.,

$$
\left[f^{n} P(f)\right]^{(k)}\left[g^{n} P(g)\right]^{(k)} \equiv p^{2}
$$


i.e.,

$$
\left[f^{n}(f-1)^{m}\right]\left[g^{n}(g-1)^{m}\right] \equiv p^{2} .
$$

If $B \neq-1$, from (4) we have

$$
\frac{1}{\mathrm{~F}} \equiv \frac{\mathrm{BG}}{(1+\mathrm{B}) \mathrm{G}-1} .
$$

Therefore

$$
\overline{\mathrm{N}}\left(r, \frac{1}{1+\mathrm{B}} ; \mathrm{G}\right)=\overline{\mathrm{N}}(r, 0 ; F) .
$$

So in view of Lemmas 1, 2 and the second fundamental theorem we get

$$
\begin{aligned}
& (n+m) T(r, g) \\
& \leq \mathrm{T}(\mathrm{r}, \mathrm{G})+\mathrm{N}_{\mathrm{k}+1}\left(\mathrm{r}, 0 ; \mathrm{g}^{\mathrm{n}} \mathrm{P}(\mathrm{g})\right)-\overline{\mathrm{N}}(\mathrm{r}, 0 ; \mathrm{G})+\mathrm{S}(\mathrm{r}, \mathrm{g}) \\
& \leq \bar{N}(r, \infty ; G)+\bar{N}(r, 0 ; G)+\bar{N}\left(r, \frac{1}{1+B} ; G\right)+N_{k+1}\left(r .0 ; g^{n} P(g)\right) \\
& -\bar{N}(r, 0 ; G)+S(r, g) \\
& \leq \bar{N}(r, \infty ; g)+(k+1) \bar{N}(r, 0 ; g)+T(r, P(g))+\bar{N}(r, 0 ; F)+S(r, g) \\
& \leq \overline{\mathrm{N}}(\mathrm{r}, \infty ; g)+(\mathrm{k}+1) \overline{\mathrm{N}}(\mathrm{r}, 0 ; g)+\mathrm{T}(\mathrm{r}, \mathrm{P}(\mathrm{g}))+(\mathrm{k}+1) \overline{\mathrm{N}}(\mathrm{r}, 0 ; f)+\mathrm{T}(\mathrm{r}, \mathrm{P}(\mathrm{f})) \\
& +k \bar{N}(r, \infty ; f)+S(r, f)+S(r, g) \\
& \leq(k+2+m) T(r, g)+(2 k+1+m) T(r, f)+S(r, f)+S(r, g) \text {. }
\end{aligned}
$$

So for $r \in I$ we have

$$
(n-3 k-3-m) T(r, g) \leq S(r, g),
$$

which is a contradiction since $n>3 k+3+m$.

Case 3. Let $B=0$. From (4) we obtain

$$
F \equiv \frac{G+A-1}{A} .
$$

If $A \neq 1$, then from (5) we obtain

$$
\bar{N}(r, 1-A ; G)=\bar{N}(r, 0 ; F) .
$$

We can similarly deduce a contradiction as in Case 2. Therefore $A=1$ and from (5) we obtain

$$
\mathrm{F} \equiv \mathrm{G},
$$


i.e.,

$$
\left[f^{n} P(f)\right]^{(k)} \equiv\left[g^{n} P(g)\right]^{(k)} .
$$

Then by Lemma 8 we have

$$
f^{n} P(f) \equiv g^{n} P(g)
$$

i.e.,

$$
f^{n}(f-1)^{m} \equiv g^{n}(g-1)^{m} .
$$

Lemma 11 Let $\mathrm{f}, \mathrm{g}$ be two transcendental meromorphic functions, $\mathrm{p}(z)$ be a non-zero polynomial with $\operatorname{deg}(\mathrm{p}(z))=\mathrm{l}, \mathrm{n}, \mathrm{k}$ be two positive integers. Let $\left[\mathrm{f}^{\mathrm{n}}\right]^{(\mathrm{k})}-\mathrm{p}$ and $\left[\mathrm{g}^{\mathrm{n}}\right]^{(\mathrm{k})}-\mathrm{p}$ share $(0, \infty)$. Suppose $\left[\mathrm{f}^{\mathrm{n}}\right]^{(\mathrm{k})}\left[\mathrm{g}^{\mathrm{n}}\right]^{(\mathrm{k})} \equiv \mathrm{p}^{2}$,

(i) if $\mathrm{p}(z)$ is not a constant and $\mathrm{n}>2 \mathrm{k}+2 \mathrm{l}-1$, then $\mathrm{f}(z)=\mathrm{c}_{1} \mathrm{e}^{\mathrm{cQ}(z)}$, $\mathrm{g}(z)=\mathrm{c}_{2} \mathrm{e}^{-\mathrm{cQ}(z)}$, where $\mathrm{Q}(z)=\int_{0}^{z} \mathrm{p}(z) \mathrm{d} z, \mathrm{c}_{1}, \mathrm{c}_{2}$ and $\mathrm{c}$ are constants such that $(\mathrm{nc})^{2}\left(\mathrm{c}_{1} \mathrm{c}_{2}\right)^{\mathrm{n}}=-1$,

(ii) if $\mathrm{p}(z)$ is a nonzero constant $\mathrm{b}$ and $\mathrm{n}>2 \mathrm{k}$, then $\mathrm{f}(z)=\mathrm{c}_{3} \mathrm{e}^{\mathrm{cz}}, \mathrm{g}(z)=$ $\mathrm{c}_{4} \mathrm{e}^{-\mathrm{c} z}$, where $\mathrm{c}_{3}, \mathrm{c}_{4}$ and $\mathrm{c}$ are constants such that $(-1)^{\mathrm{k}}\left(\mathrm{c}_{3} \mathrm{c}_{4}\right)^{\mathrm{n}}(\mathrm{nc})^{2 \mathrm{k}}=$ $b^{2}$.

Proof. Suppose

$$
\left[\mathrm{f}^{\mathrm{n}}\right]^{(\mathrm{k})}\left[\mathrm{g}^{\mathrm{n}}\right]^{(\mathrm{k})} \equiv \mathrm{p}^{2} .
$$

We consider the following cases.

Case 1: Let $\operatorname{deg}(p(z))=l(\geq 1)$.

Let $z_{0}$ be a zero of $f$ with multiplicity $q$. Then $z_{0}$ be a zero of $\left[f^{n}\right]^{(k)}$ with multiplicity $n q-k$. Now one of the following possibilities holds.

(i) $z_{0}$ will be neither a zero of $\left[\mathrm{g}^{\mathrm{n}}\right]^{(\mathrm{k})}$ nor a pole of $\mathrm{g}$; (ii) $z_{0}$ will be a zero of $\mathrm{g}$; (iii) $z_{0}$ will be a zero of $\left[\mathrm{g}^{\mathrm{n}}\right]^{(\mathrm{k})}$ but not a zero of $\mathrm{g}$ and (iv) $z_{0}$ will be a pole of $\mathrm{g}$.

We now explain only the above two possibilities (i) and (iv) because other two possibilities follow from these.

For the possibility (i): Note that since $n \geq 2 k+2 l$, we must have

$$
n q-k \geq n-k \geq k+2 l
$$


Thus $z_{0}$ must be a zero of $\left[\mathrm{f}^{\mathrm{n}}\right]^{(\mathrm{k})}$ with multiplicity at least $k+2 l$. But we see from $(7)$ that $z_{0}$ must be a zero of $p^{2}(z)$ with multiplicity atmost $2 l$. Hence we arrive at a contradiction and so $f$ has no zero in this case.

For the possibility (iv): Let $z_{0}$ be a pole of $g$ with multiplicity $q_{1}$. Clearly $z_{0}$ will be pole of $\left[g^{n}\right]^{(k)}$ with multiplicity $n q_{1}+k$. Obviously $q>q_{1}$, or else $z_{0}$ is a pole of $p(z)$, which is a contradiction since $p(z)$ is a polynomial. Clearly $n q-k \geq n q_{1}+k$. Now

$$
n q-k=n q_{1}+k
$$

implies that

$$
\mathrm{n}\left(\mathrm{q}-\mathrm{q}_{1}\right)=2 \mathrm{k} .
$$

Since $n \geq 2 k+2 l$, we get a contradiction from (9). Hence we must have

$$
n q-k>n q_{1}+k .
$$

This shows that $z_{0}$ is a zero of $p(z)$ and we have $N(r, 0 ; f)=O(\log r)$. Similarly we can prove that $\mathrm{N}(r, 0 ; g)=\mathrm{O}(\log r)$. Thus in general we can take $\mathrm{N}(r, 0 ; f)+$ $\mathrm{N}(\mathrm{r}, 0 ; \mathrm{g})=\mathrm{O}(\log \mathrm{r})$.

We know that

$$
N\left(r, \infty ;\left[f^{\mathfrak{n}}\right]^{(k)}\right)=n N(r, \infty ; f)+k \bar{N}(r, \infty ; f) .
$$

Also by Lemma 7 we have

$$
\begin{aligned}
\mathrm{N}\left(r, 0 ;\left[g^{n}\right]^{(k)}\right) & \leq n N(r, 0 ; g)+k \bar{N}(r, \infty ; g)+S(r, g) \\
& \leq k \bar{N}(r, \infty ; g)+O(\log r)+S(r, g) .
\end{aligned}
$$

From (7) we get

$$
N\left(r, \infty ;\left[f^{n}\right]^{(k)}\right)=N\left(r, 0 ;\left[g^{n}\right]^{(k)}\right),
$$

i.e.,

$$
n N(r, \infty ; f)+k \bar{N}(r, \infty ; f) \leq k \bar{N}(r, \infty ; g)+O(\log r)+S(r, g) .
$$

Similarly we get

$$
n \mathrm{~N}(r, \infty ; g)+k \bar{N}(r, \infty ; g) \leq k \bar{N}(r, \infty ; f)+O(\log r)+S(r, f) .
$$

Since $f$ and $g$ are transcendental, it follows that

$$
S(r, f)+O(\log r)=S(r, f), \quad S(r, g)+O(\log r)=S(r, g) .
$$


Combining (10) and (11) we get

$$
N(r, \infty ; f)+N(r, \infty ; g)=S(r, f)+S(r, g) .
$$

By Lemma 9 we have $S(r, f)=S(r, g)$ and so we obtain

$$
N(r, \infty ; f)=S(r, f), \quad N(r, \infty ; g)=S(r, g)
$$

Let

$$
F_{1}=\frac{\left[f^{n}\right]^{(k)}}{p}, \quad G_{1}=\frac{\left[g^{n}\right]^{(k)}}{p} .
$$

Note that $T\left(r, F_{1}\right) \leq n(k+1) T(r, f)+S(r, f)$ and so $T\left(r, F_{1}\right)=O(T(r, f))$. Also by Lemma 2 one can obtain $T(r, f)=O\left(T\left(r, F_{1}\right)\right)$. Hence $S\left(r, F_{1}\right)=S(r, f)$. Similarly we get $S\left(r, G_{1}\right)=S(r, g)$. Also

$$
\mathrm{F}_{1} \mathrm{G}_{1} \equiv 1 .
$$

If $F_{1} \equiv c G_{1}$, where $c$ is a nonzero constant, then $F_{1}$ is a constant and so $f$ is a polynomial, which contradicts our assumption. Hence $F_{1} \not \equiv c G_{1}$ and so in view of $(14)$ we see that $F_{1}$ and $G_{1}$ share $(-1,0)$.

Now by Lemma 7 we have

$$
N\left(r, 0 ; F_{1}\right) \leq n N(r, 0 ; f)+k \bar{N}(r, \infty ; f)+S(r, f) \leq S\left(r, F_{1}\right) .
$$

Similarly we have

$$
\mathrm{N}\left(r, 0 ; \mathrm{G}_{1}\right) \leq \mathrm{nN}(\mathrm{r}, 0 ; g)+\mathrm{k} \overline{\mathrm{N}}(\mathrm{r}, \infty ; \mathrm{g})+\mathrm{S}(\mathrm{r}, \mathrm{g}) \leq \mathrm{S}\left(\mathrm{r}, \mathrm{G}_{1}\right)
$$

Also we see that

$$
N\left(r, \infty ; F_{1}\right)=S\left(r, F_{1}\right), \quad N\left(r, \infty ; G_{1}\right)=S\left(r, G_{1}\right) .
$$

Here it is clear that $T\left(r, F_{1}\right)=T\left(r, G_{1}\right)+O(1)$. Let

$$
f_{1}=\frac{F_{1}}{G_{1}} .
$$

and

$$
f_{2}=\frac{F_{1}-1}{G_{1}-1} .
$$


Clearly $f_{1}$ is non-constant. If $f_{2}$ is a nonzero constant then $F_{1}$ and $G_{1}$ share $(\infty, \infty)$ and so from (14) we conclude that $F_{1}$ and $G_{1}$ have no poles. Next we suppose that $f_{2}$ is non-constant. Also we see that

$$
F_{1}=\frac{f_{1}\left(1-f_{2}\right)}{f_{1}-f_{2}}, \quad G_{1}=\frac{1-f_{2}}{f_{1}-f_{2}} .
$$

Clearly

$$
T\left(r, F_{1}\right) \leq 2\left[T\left(r, f_{1}\right)+T\left(r, f_{2}\right)\right]+O(1)
$$

and

$$
\mathrm{T}\left(r, f_{1}\right)+\mathrm{T}\left(r, f_{2}\right) \leq 4 \mathrm{~T}\left(\mathrm{r}, \mathrm{F}_{1}\right)+\mathrm{O}(1) .
$$

These give $S\left(r, F_{1}\right)=S\left(r ; f_{1}, f_{2}\right)$. Also we see that

$$
\bar{N}\left(r, 0 ; f_{i}\right)+\bar{N}\left(r, \infty ; f_{i}\right)=S\left(r ; f_{1}, f_{2}\right)
$$

for $i=1,2$.

Next we suppose $\bar{N}\left(r,-1 ; F_{1}\right) \neq S\left(r, F_{1}\right)$, otherwise $F_{1}$ will be a constant. Also we see that

$$
\overline{\mathrm{N}}\left(r,-1 ; F_{1}\right) \leq \mathrm{N}_{0}\left(r, 1 ; f_{1}, f_{2}\right) .
$$

Thus we have

$$
T\left(r, f_{1}\right)+T\left(r, f_{2}\right) \leq 4 N_{0}\left(r, 1 ; f_{1}, f_{2}\right)+S\left(r, F_{1}\right) .
$$

Then by Lemma 4 there exist two integers $s$ and $\mathbf{t}(|\mathbf{s}|+|\mathbf{t}|>0)$ such that

$$
f_{1}^{s} f_{2}^{t} \equiv 1
$$

i.e.,

$$
\left[\frac{F_{1}}{G_{1}}\right]^{s}\left[\frac{F_{1}-1}{G_{1}-1}\right]^{t} \equiv 1
$$

We now consider following cases.

Case (i) Let $s=0$ and $t \neq 0$. Then from (15) we get

$$
\left(F_{1}-1\right)^{t} \equiv\left(G_{1}-1\right)^{t} .
$$

This shows that $F_{1}$ and $G_{1}$ share $(\infty, \infty)$ and so from (14) we conclude that $F_{1}$ and $G_{1}$ have no poles.

Case (ii) Suppose $s \neq 0$ and $t=0$. Then from (15) we get

$$
\mathrm{F}_{1}^{\mathrm{s}} \equiv \mathrm{G}_{1}^{\mathrm{s}}
$$


and so we arrive at a contradiction from (14).

Case (iii): Suppose $s>0$ and $t=-t_{1}$, where $t_{1}>0$. Then we have

$$
\left[\frac{F_{1}}{G_{1}}\right]^{s} \equiv\left[\frac{F_{1}-1}{G_{1}-1}\right]^{t_{1}} \text {. }
$$

If possible suppose $F_{1}$ has a pole. Let $z_{p_{1}}$ be a pole of $F_{1}$ of multiplicity $p_{1}$. Then from (14) we see that $z_{p_{1}}$ must be a zero of $G_{1}$ of multiplicity $p_{1}$. Now from (16) we get $2 s=t_{1}$ and so

$$
\left[\frac{F_{1}}{G_{1}}\right]^{s} \equiv\left[\frac{F_{1}-1}{G_{1}-1}\right]^{2 s}
$$

This implies that

$F_{1}^{s-1}+F_{1}^{s-2} G_{1}+F_{1}^{s-3} G_{1}^{2}+\ldots+F_{1} G_{1}^{s-2}+G_{1}^{s-1} \equiv G_{1}^{s} \frac{\left(F_{1}-1\right)^{2 s}-\left(G_{1}-1\right)^{2 s}}{\left(G_{1}-1\right)^{2 s}\left(F_{1}-G_{1}\right)}$

If $z_{p}$ is a zero of $F_{1}-1$ with multiplicity $p$ then the Taylor expansion of $F_{1}-1$ about $z_{p}$ is

$$
\mathrm{F}_{1}-1=\mathrm{a}_{\mathfrak{p}}\left(z-z_{\mathfrak{p}}\right)^{\mathfrak{p}}+\mathrm{a}_{\mathfrak{p}+1}\left(z-z_{\mathfrak{p}}\right)^{p+1}+\ldots \ldots, \quad \mathrm{a}_{\mathfrak{p}} \neq 0 .
$$

Since $F_{1}-1$ and $G_{1}-1$ share $(0, \infty)$,

$$
\mathrm{G}_{1}-1=\mathrm{b}_{\mathrm{p}}\left(z-z_{\mathrm{p}}\right)^{\mathrm{p}}+\mathrm{b}_{\mathrm{p}+1}\left(z-z_{\mathrm{p}}\right)^{\mathrm{p}+1}+\ldots, \quad \mathrm{b}_{\mathfrak{p}} \neq 0 .
$$

Let

$$
\Phi_{1}=\frac{F_{1}^{\prime}}{F_{1}}-\frac{G_{1}^{\prime}}{G_{1}} \text { and } \Phi_{2}=\left(\frac{F_{1}^{\prime}}{F_{1}}\right)^{2 s}-\left(\frac{G_{1}^{\prime}}{G_{1}}\right)^{2 s} .
$$

Since $F_{1} \not \equiv c G_{1}$, where $c$ is a nonzero constant, it follows that $\Phi_{1} \not \equiv 0$ and $\Phi_{2} \not \equiv 0$. Also

$$
\mathrm{T}\left(\mathrm{r}, \Phi_{1}\right)=\mathrm{S}\left(\mathrm{r}, \mathrm{F}_{1}\right) \text { and } \mathrm{T}\left(\mathrm{r}, \Phi_{2}\right)=\mathrm{S}\left(\mathrm{r}, \mathrm{F}_{1}\right) .
$$

From (18) we find

$$
\bar{N}_{(2}\left(r, 1 ; F_{1}\right)=\bar{N}_{(2}\left(r, 1 ; G_{1}\right) \leq N\left(r, 0 ; \Phi_{1}\right)=S\left(r, F_{1}\right) .
$$

Let $p=1$. If $a_{1}=b_{1}$, then by an elementary calculation gives that $\Phi_{1}(z)=$ $\mathrm{O}\left(\left(z-z_{1}\right)^{\mathrm{k}}\right)$, where $\mathrm{k}$ is a positive integer. This proves that $z_{1}$ is a zero of $\Phi_{1}$. Next we suppose $a_{1} \neq b_{1}$, but $a_{1}^{2 s}=b_{1}^{2 s}$. Then by an elementary calculation we get $\Phi_{2}(z)=\mathrm{O}\left(\left(z-z_{1}\right)^{\mathrm{q}}\right)$ where $\mathrm{q}$ is a positive integer. This proves that $z_{1}$ 
is a zero of $\Phi_{2}$.

Finally we suppose $a_{1} \neq b_{1}$ and $a_{1}^{2 s} \neq b_{1}^{2 s}$. Therefore from (17) we arrive at a contradiction. Hence

$$
\left.N_{1)}\left(r, 1 ; F_{1}\right)=N_{1}\right)\left(r, 1 ; G_{1}\right)=S\left(r, F_{1}\right) .
$$

But this is impossible as $\bar{N}\left(r, 1 ; F_{1}\right) \sim T\left(r, F_{1}\right)$ and $\bar{N}\left(r, 1 ; G_{1}\right) \sim T\left(r, G_{1}\right)$. Hence $F_{1}$ has no pole. Similarly we can prove that $G_{1}$ also has no poles.

Case (iv): Suppose either $s>0$ and $t>0$ or $s<0$ and $t<0$. Then from (15) one can easily prove that $F_{1}$ and $G_{1}$ have no poles. Consequently from (14) we see that $F_{1}$ and $G_{1}$ have no zeros. We deduce from (13) that both $f$ and $g$ have no pole.

Since $F_{1}$ and $G_{1}$ have no zeros and poles, we have

$$
F_{1} \equiv e^{\gamma_{1}} G_{1}
$$

i.e.,

$$
\left[\mathrm{f}^{\mathfrak{n}}\right]^{(\mathrm{k})} \equiv e^{\gamma_{1}}\left[\mathrm{~g}^{\mathrm{n}}\right]^{(\mathrm{k})},
$$

where $\gamma_{1}$ is a non-constant entire function. Then from (7) we get

$$
\left[\mathrm{f}^{\mathrm{n}}\right]^{(\mathrm{k})} \equiv c e^{\frac{1}{2} \gamma_{1}} p, \quad\left[\mathrm{~g}^{\mathrm{n}}\right]^{(\mathrm{k})} \equiv c e^{-\frac{1}{2} \gamma_{1}} \mathrm{p},
$$

where $c= \pm 1$. Since $N(r, 0 ; f)=O(\log r)$ and $N(r, 0 ; g)=O(\log r)$, so we can take

$$
f(z)=P_{1}(z) e^{\alpha_{1}(z)}, \quad g(z)=Q_{1}(z) e^{\beta_{1}(z)},
$$

$P_{1}, Q_{1}$ are nonzero polynomials, $\alpha_{1}, \beta_{1}$ are two non-constant entire functions. If possible suppose that $P_{1}(z)$ is not a constant. Let $z_{1}$ be a zero of $f$ with multiplicity $t$. Then $z_{1}$ must be a zero of $\left[\mathrm{f}^{\mathrm{n}}\right]^{(\mathrm{k})}$ with multiplicity $n t-k$. Note that $n t-k \geq n-k \geq k+2 l$, as $n \geq 2 k+2 l$. Clearly $z_{1}$ must be a zero of $p^{2}(z)$ with multiplicity at least $k+2 l$, which is impossible since $z_{1}$ can be a zero of $p^{2}(z)$ with multiplicity at most $2 l$. Hence $P_{1}(z)$ is a constant. Similarly we can prove that $Q_{1}(z)$ is a constant. So we can rewrite $f$ and $g$ as follows

$$
f=e^{\alpha}, \quad g=e^{\beta} .
$$

We deduce from (7) and (21) that either both $\alpha$ and $\beta$ are transcendental entire functions or both $\alpha$ and $\beta$ are polynomials. We now consider following cases.

Subcase 1.1: Let $k \geq 2$. 
First we suppose both $\alpha$ and $\beta$ are transcendental entire functions.

Note that

$$
S(r, n \alpha)=S\left(r, \frac{\left[f^{n}\right]^{\prime}}{f^{n}}\right), \quad S(r, n \beta)=S\left(r, \frac{\left[g^{n}\right]^{\prime}}{g^{n}}\right) .
$$

Moreover we see that

$$
\begin{aligned}
& N\left(r, 0 ;\left[f^{n}\right]^{(k)}\right) \leq N\left(r, 0 ; p^{2}\right)=O(\log r) . \\
& N\left(r, 0 ;\left[g^{n}\right]^{(k)}\right) \leq N\left(r, 0 ; p^{2}\right)=O(\log r) .
\end{aligned}
$$

From these and using (21) we have

$$
N\left(r, \infty ; f^{n}\right)+N\left(r, 0 ; f^{n}\right)+N\left(r, 0 ;\left[f^{n}\right]^{(k)}\right)=S(r, n \alpha)=S\left(r, \frac{\left[f^{\mathfrak{n}}\right]^{\prime}}{f^{n}}\right)
$$

and

$$
N\left(r, \infty ; g^{n}\right)+N\left(r, 0 ; g^{n}\right)+N\left(r, 0 ;\left[g^{n}\right]^{(k)}\right)=S(r, n \beta)=S\left(r, \frac{\left[g^{n}\right]^{\prime}}{g^{n}}\right) .
$$

Then from (22), (23) and Lemma 5 we must have

$$
f(z)=e^{\mathrm{a} z+\mathrm{b}}, \quad g(z)=e^{\mathrm{c} z+\mathrm{d}},
$$

where $a \neq 0, b, c \neq 0$ and $d$ are constants. But these types of $f$ and $g$ do not agree with the relation (7).

Next we suppose $\alpha$ and $\beta$ are both polynomials.

Clearly $\alpha+\beta \equiv C$ and $\operatorname{deg}(\alpha)=\operatorname{deg}(\beta)$. Also $\alpha^{\prime} \equiv \beta^{\prime}$. If $\operatorname{deg}(\alpha)=\operatorname{deg}(\beta)=$ 1 , then we again get a contradiction from $(7)$.

Next we suppose $\operatorname{deg}(\alpha)=\operatorname{deg}(\beta) \geq 2$.

We deduce from (21) that

$$
\begin{aligned}
& \left(f^{n}\right)^{\prime}=n \alpha^{\prime} e^{n \alpha} \\
& \left(f^{n}\right)^{\prime \prime}=\left[n^{2}\left(\alpha^{\prime}\right)^{2}+n \alpha^{\prime \prime}\right] e^{n \alpha} \\
& \left(f^{n}\right)^{\prime \prime \prime}=\left[n^{3}\left(\alpha^{\prime}\right)^{3}+3 n^{2} \alpha^{\prime} \alpha^{\prime \prime}+n \alpha^{\prime \prime \prime}\right] e^{n \alpha} \\
& \left(f^{n}\right)^{(i v)}=\left[n^{4}\left(\alpha^{\prime}\right)^{4}+6 n^{3}\left(\alpha^{\prime}\right)^{2} \alpha^{\prime \prime}+3 n^{2}\left(\alpha^{\prime \prime}\right)^{2}+4 n^{2} \alpha^{\prime} \alpha^{\prime \prime \prime}+n \alpha^{(i v)}\right] e^{n \alpha} \\
& \left(f^{n}\right)^{(v)}=\left[n^{5}\left(\alpha^{\prime}\right)^{5}+10 n^{4}\left(\alpha^{\prime}\right)^{3} \alpha^{\prime \prime}+15 n^{3} \alpha^{\prime}\left(\alpha^{\prime \prime}\right)^{2}+10 n^{3}\left(\alpha^{\prime}\right)^{2}\right. \\
& \left.\alpha^{\prime \prime \prime}+10 n^{2} \alpha^{\prime \prime} \alpha^{\prime \prime \prime}+5 n^{2} \alpha^{\prime} \alpha^{(i v)}+n \alpha^{(v)}\right] e^{n \alpha} \\
& \cdots \quad \cdots \quad \cdots \quad \cdots \quad \cdots \quad \cdots \quad \cdots \\
& {\left[f^{n}\right]^{(k)}=\left[n^{k}\left(\alpha^{\prime}\right)^{k}+K\left(\alpha^{\prime}\right)^{k-2} \alpha^{\prime \prime}+P_{k-2}\left(\alpha^{\prime}\right)\right] e^{n \alpha},}
\end{aligned}
$$


where $K$ is a suitably positive integer and $P_{k-2}\left(\alpha^{\prime}\right)$ is a differential polynomial in $\alpha^{\prime}$.

Similarly we get

$$
\begin{aligned}
{\left[g^{n}\right]^{(k)} } & =\left[n^{k}\left(\beta^{\prime}\right)^{k}+K\left(\beta^{\prime}\right)^{k-2} \beta^{\prime \prime}+P_{k-2}\left(\beta^{\prime}\right)\right] e^{n \beta} \\
& =\left[(-1)^{k} n^{k}\left(\alpha^{\prime}\right)^{k}-K(-1)^{k-2}\left(\alpha^{\prime}\right)^{k-2} \alpha^{\prime \prime}+P_{k-2}\left(-\alpha^{\prime}\right)\right] e^{n \beta} .
\end{aligned}
$$

Since $\operatorname{deg}(\alpha) \geq 2$, we observe that $\operatorname{deg}\left(\left(\alpha^{\prime}\right)^{k}\right) \geq k \operatorname{deg}\left(\alpha^{\prime}\right)$ and so $\left(\alpha^{\prime}\right)^{k-2} \alpha^{\prime \prime}$ is either a nonzero constant or $\operatorname{deg}\left(\left(\alpha^{\prime}\right)^{k-2} \alpha^{\prime \prime}\right) \geq(k-1) \operatorname{deg}\left(\alpha^{\prime}\right)-1$. Also we see that

$\operatorname{deg}\left(\left(\alpha^{\prime}\right)^{k}\right)>\operatorname{deg}\left(\left(\alpha^{\prime}\right)^{k-2} \alpha^{\prime \prime}\right)>\operatorname{deg}\left(P_{k-2}\left(\alpha^{\prime}\right)\right)\left(\right.$ or $\left.\operatorname{deg}\left(P_{k-2}\left(-\alpha^{\prime}\right)\right)\right)$.

From (19), it is clear that the polynomials

$$
n^{k}\left(\alpha^{\prime}\right)^{k}+K\left(\alpha^{\prime}\right)^{k-2} \alpha^{\prime \prime}+P_{k-2}\left(\alpha^{\prime}\right)
$$

and

$$
(-1)^{k} n^{k}\left(\alpha^{\prime}\right)^{k}-K(-1)^{k-2}\left(\alpha^{\prime}\right)^{k-2} \alpha^{\prime \prime}+P_{k-2}\left(-\alpha^{\prime}\right)
$$

must be identical but this is impossible for $k \geq 2$. Actually the terms $n^{k}\left(\alpha^{\prime}\right)^{k}+$ $\mathrm{K}\left(\alpha^{\prime}\right)^{\mathrm{k}-2} \alpha^{\prime \prime}$ and $(-1)^{\mathrm{k}} \mathrm{n}^{\mathrm{k}}\left(\alpha^{\prime}\right)^{\mathrm{k}}-\mathrm{K}(-1)^{\mathrm{k}-2}\left(\alpha^{\prime}\right)^{\mathrm{k}-2} \alpha^{\prime \prime}$ can not be identical for $k \geq 2$.

Subcase 2: Let $k=1$. Then from (7) we get

$$
A B \alpha^{\prime} \beta^{\prime} e^{n(\alpha+\beta)} \equiv p^{2},
$$

where $A B=n^{2}$. Let $\alpha+\beta=\gamma$. Suppose that $\alpha$ and $\beta$ are both transcendental entire functions. From (25) we know that $\gamma$ is not a constant since in that case we get a contradiction. Then from (25) we get

$$
\operatorname{AB} \alpha^{\prime}\left(\gamma^{\prime}-\alpha^{\prime}\right) e^{n \gamma} \equiv p^{2} .
$$

We have $T\left(r, \gamma^{\prime}\right)=m\left(r, \gamma^{\prime}\right) \leq m\left(r, \frac{\left(e^{n \gamma}\right)^{\prime}}{e^{n \gamma}}\right)+O(1)=S\left(r, e^{n \gamma \gamma}\right)$. Thus from (26) we get

$$
\begin{aligned}
\mathrm{T}\left(\mathrm{r}, e^{\mathrm{n} \gamma}\right) & \leq \mathrm{T}\left(\mathrm{r}, \frac{\mathrm{p}^{2}}{\alpha^{\prime}\left(\gamma^{\prime}-\alpha^{\prime}\right)}\right)+\mathrm{O}(1) \\
& \leq \mathrm{T}\left(\mathrm{r}, \alpha^{\prime}\right)+\mathrm{T}\left(\mathrm{r}, \gamma^{\prime}-\alpha^{\prime}\right)+\mathrm{O}(\log r)+\mathrm{O}(1) \\
& \leq 2 \mathrm{~T}\left(\mathrm{r}, \alpha^{\prime}\right)+\mathrm{S}\left(\mathrm{r}, \alpha^{\prime}\right)+\mathrm{S}\left(\mathrm{r}, \mathrm{e}^{\mathrm{n} \gamma}\right),
\end{aligned}
$$


which implies that $T\left(r, e^{\mathfrak{n} \gamma}\right)=\mathrm{O}\left(\mathrm{T}\left(\mathrm{r}, \alpha^{\prime}\right)\right)$ and so $\mathrm{S}\left(\mathrm{r}, \mathrm{e}^{\mathrm{n} \gamma}\right)$ can be replaced by $S\left(r, \alpha^{\prime}\right)$. Thus we get $T\left(r, \gamma^{\prime}\right)=S\left(r, \alpha^{\prime}\right)$ and so $\gamma^{\prime}$ is a small function with respect to $\alpha^{\prime}$. In view of (26) and by the second fundamental theorem for small functions we get

$$
\begin{aligned}
\mathrm{T}\left(\mathrm{r}, \alpha^{\prime}\right) & \leq \overline{\mathrm{N}}\left(\mathrm{r}, \infty ; \alpha^{\prime}\right)+\overline{\mathrm{N}}\left(\mathrm{r}, 0 ; \alpha^{\prime}\right)+\overline{\mathrm{N}}\left(\mathrm{r}, 0 ; \alpha^{\prime}-\gamma^{\prime}\right)+\mathrm{S}\left(\mathrm{r}, \alpha^{\prime}\right) \\
& \leq \mathrm{O}(\log r)+\mathrm{S}\left(\mathrm{r}, \alpha^{\prime}\right),
\end{aligned}
$$

which shows that $\alpha^{\prime}$ is a polynomial and so $\alpha$ is a polynomial, which contradicts that $\alpha$ is a transcendental entire function. Next suppose without loss of generality that $\alpha$ is a polynomial and $\beta$ is a transcendental entire function. Thus $\gamma$ is transcendental. So in view of (26) we can obtain

$$
\begin{aligned}
\mathrm{nT}\left(\mathrm{r}, e^{\gamma}\right) & \leq \mathrm{T}\left(\mathrm{r}, \frac{\mathrm{p}^{2}}{\alpha^{\prime}\left(\gamma^{\prime}-\alpha^{\prime}\right)}\right)+\mathrm{O}(1) \\
& \leq \mathrm{T}\left(\mathrm{r}, \alpha^{\prime}\right)+\mathrm{T}\left(\mathrm{r}, \gamma^{\prime}-\alpha^{\prime}\right)+\mathrm{S}\left(\mathrm{r}, \mathrm{e}^{\gamma}\right) \\
& \leq \mathrm{T}\left(\mathrm{r}, \gamma^{\prime}\right)+\mathrm{S}\left(\mathrm{r}, \mathrm{e}^{\gamma}\right)=\mathrm{S}\left(\mathrm{r}, \mathrm{e}^{\gamma}\right),
\end{aligned}
$$

which leads a contradiction. Thus $\alpha$ and $\beta$ are both polynomials. Also from (25) we can conclude that $\alpha+\beta \equiv C$ for a constant $C$ and so $\alpha^{\prime}+\beta^{\prime} \equiv 0$. Again from (25) we get $n^{2} e^{n C} \alpha^{\prime} \beta^{\prime} \equiv p^{2}$. By computation we get

$$
\alpha^{\prime}=c p, \quad \beta^{\prime}=-c p .
$$

Hence

$$
\alpha=c Q+b_{1}, \quad \beta=-c Q+b_{2},
$$

where $Q(z)=\int_{0}^{z} p(z) d z$ and $b_{1}, b_{2}$ are constants. Finally $f$ and $g$ take the form

$$
f(z)=c_{1} e^{c Q(z)}, \quad g(z)=c_{2} e^{-c Q(z)},
$$

where $c_{1}, c_{2}$ and $c$ are constants such that $(n c)^{2}\left(c_{1} c_{2}\right)^{n}=-1$.

Case 2: Let $p(z)$ be a nonzero constant $b$. Since $n>2 k$, one can easily prove that $f$ and $g$ have no zeros. Now proceeding in the same way as done in proof of Case 1 we get $f=e^{\alpha}$ and $g=e^{\beta}$, where $\alpha$ and $\beta$ are two non-constant entire functions.

We now consider following two subcases:

Subcase 2.1: Let $k \geq 2$.

We see that $\mathrm{f}^{\mathrm{n}}(z)\left[\mathrm{f}^{\mathrm{n}}(z)\right]^{(\mathrm{k})} \neq 0$ and $\mathrm{g}^{\mathrm{n}}(z)\left[\mathrm{g}^{\mathrm{n}}(z)\right]^{(\mathrm{k})} \neq 0$. Then by Lemma 6 we must have

$$
f(z)=e^{a z+b}, \quad g(z)=e^{c z+d},
$$


where $a \neq 0, b, c \neq 0$ and $d$ are constants. But from (7) we see that $a+c=0$. Subcase 2.1: Let $k=1$.

Considering Subcase 1.2 one can easily get

$$
f(z)=e^{\mathrm{a} z+\mathrm{b}}, \quad g(z)=e^{\mathrm{c} z+\mathrm{d}},
$$

where $a \neq 0, b, c \neq 0$ and $d$ are constants. Finally $f$ and $g$ take the form

$$
f(z)=c_{3} e^{d z}, \quad g(z)=c_{4} e^{-d z},
$$

where $c_{3}, c_{4}$ and $d$ are nonzero constants such that $(-1)^{k}\left(c_{3} c_{4}\right)^{n}(n d)^{2 k}=b^{2}$. This completes the proof.

Lemma 12 Let $\mathrm{f}, \mathrm{g}$ be two transcendental meromorphic functions, let $\mathrm{n}, \mathrm{m}$ and $\mathrm{k}$ be three positive integers such that $\mathrm{n}>\mathrm{k}$. If $\mathrm{f}$ and $\mathrm{g}$ share $(\infty, 0)$ then $\left[\mathrm{f}^{\mathrm{n}}(\mathrm{f}-1)^{\mathrm{m}}\right]^{(\mathrm{k})}\left[\mathrm{g}^{\mathrm{n}}(\mathrm{g}-1)^{\mathrm{m}}\right]^{(\mathrm{k})} \not \equiv \mathrm{p}^{2}$, where $\mathrm{p}(\mathrm{z})$ is a non zero polynomial.

Proof. Suppose

$$
\left[f^{n}(f-1)^{m}\right]^{(k)}\left[g^{n}(g-1)^{m}\right]^{(k)} \equiv p^{2} .
$$

Since $f$ and $g$ share $(\infty, 0)$ we have from $(31)$ that $f$ and $g$ are transcendental entire functions. So we can take

$$
f(z)=h(z) e^{\alpha(z)}
$$

where $h$ is a nonzero polynomial and $\alpha$ is a non-constant entire function. We know that $(w-1)^{m}=a_{m} w^{m}+a_{m-1} w^{m-1}+\ldots+a_{0}$, where $a_{i}=$ $(-1)^{m-i}{ }^{m} C_{m-i}, i=0,1,2, \ldots, m$. Since $f=h e^{\alpha}$, then by induction we get

$$
\left(a_{i} f^{n+i}\right)^{(k)}=t_{i}\left(\alpha^{\prime}, \alpha^{\prime \prime}, \ldots, \alpha^{(k)}, h, h^{\prime}, \ldots, h^{(k)}\right) e^{(n+i) \alpha},
$$

where $t_{i}\left(\alpha^{\prime}, \alpha^{\prime \prime}, \ldots, \alpha^{(k)}, h, h^{\prime}, \ldots, h^{(k)}\right)(i=0,1,2, \ldots, m)$ are differential polynomials in $\alpha^{\prime}, \alpha^{\prime \prime}, \ldots, \alpha^{(k)}, h, h^{\prime}, \ldots, h^{(k)}$. Obviously

$$
t_{i}\left(\alpha^{\prime}, \alpha^{\prime \prime}, \ldots, \alpha^{(k)}, h, h^{\prime}, \ldots, h^{(k)}\right) \neq \equiv 0,
$$

for $i=0,1,2, \ldots, m$ and $\left[f^{n}(f-1)^{m}\right]^{(k)} \not \equiv 0$. Now from (31) and (33) we obtain

$$
\bar{N}\left(r, 0 ; t_{m} e^{m \alpha(z)}+\ldots+t_{0}\right) \leq N\left(r, 0 ; p^{2}\right)=S(r, f) .
$$


Since $\alpha$ is an entire function, we obtain $T\left(r, \alpha^{(j)}\right)=S(r, f)$ for $j=1,2, \ldots, k$. Hence $T\left(r, t_{i}\right)=S(r, f)$ for $i=0,1,2, \ldots, m$. So from (34) and using second fundamental theorem for small functions (see [17]), we obtain

$$
\begin{aligned}
\mathrm{mT}(r, f)= & T\left(r, t_{m} e^{m \alpha}+\ldots+t_{1} e^{\alpha}\right)+S(r, f) \\
\leq & \bar{N}\left(r, 0 ; t_{m} e^{m \alpha}+\ldots+t_{1} e^{\alpha}\right)+\bar{N}\left(r, 0 ; t_{m} e^{m \alpha}+\ldots+t_{1} e^{\alpha}+t_{0}\right) \\
& +S(r, f) \\
\leq & \bar{N}\left(r, 0 ; t_{m} e^{(m-1) \alpha}+\ldots+t_{1}\right)+S(r, f) \\
\leq & (m-1) T(r, f)+S(r, f),
\end{aligned}
$$

which is a contradiction. This completes the Lemma.

Lemma 13 Let $\mathrm{f}$ and $\mathrm{g}$ be two non-constant meromorphic functions and $\alpha(\not \equiv$ $0, \infty)$ be small function of $\mathrm{f}$ and $\mathrm{g}$. Let $\mathrm{n}, \mathrm{m}$ and $\mathrm{k}$ be three positive integers such that $\mathrm{n} \geq \mathrm{m}+3$. Then

$$
\left[f^{n}(f-1)^{m}\right]^{(k)}\left[g^{n}(g-1)^{m}\right]^{(k)} \not \equiv \alpha^{2}, \text { for } k=1 .
$$

Proof. We omit the proof since it can be proved in the line of the proof of Lemma 3 [14].

Lemma 14 [1] If $\mathrm{f}, \mathrm{g}$ be two non-constant meromorphic functions such that they share $(1,1)$. Then

$$
2 \bar{N}_{L}(r, 1 ; f)+2 \bar{N}_{L}(r, 1 ; g)+\bar{N}_{E}^{(2}(r, 1 ; f)-\bar{N}_{f>2}(r, 1 ; g) \leq N(r, 1 ; g)-\bar{N}(r, 1 ; g) .
$$

Lemma 15 [2] Let $\mathrm{f}, \mathrm{g}$ share $(1,1)$. Then

$$
\bar{N}_{f>2}(r, 1 ; g) \leq \frac{1}{2} \bar{N}(r, 0 ; f)+\frac{1}{2} \bar{N}(r, \infty ; f)-\frac{1}{2} N_{0}\left(r, 0 ; f^{\prime}\right)+S(r, f),
$$

where $\mathrm{N}_{0}\left(\mathrm{r}, 0 ; \mathrm{f}^{\prime}\right)$ is the counting function of those zeros of $\mathrm{f}^{\prime}$ which are not the zeros of $\mathrm{f}(\mathrm{f}-1)$.

Lemma 16 [2] Let $\mathrm{f}$ and $\mathrm{g}$ be two non-constant meromorphic functions sharing $(1,0)$. Then

$$
\begin{aligned}
& \bar{N}_{L}(r, 1 ; f)+2 \bar{N}_{L}(r, 1 ; g)+\bar{N}_{E}^{(2}(r, 1 ; f)-\bar{N}_{f>1}(r, 1 ; g)-\bar{N}_{g>1}(r, 1 ; f) \\
\leq & N(r, 1 ; g)-\bar{N}(r, 1 ; g) .
\end{aligned}
$$

Lemma 17 [2] Let $\mathrm{f}, \mathrm{g}$ share $(1,0)$. Then

$$
\overline{\mathrm{N}}_{\mathrm{L}}(\mathrm{r}, 1 ; f) \leq \overline{\mathrm{N}}(r, 0 ; f)+\overline{\mathrm{N}}(\mathrm{r}, \infty ; f)+\mathrm{S}(\mathrm{r}, \mathrm{f})
$$


Lemma 18 [2] Let $\mathrm{f}, \mathrm{g}$ share $(1,0)$. Then
(i) $\bar{N}_{f>1}(r, 1 ; g) \leq \bar{N}(r, 0 ; f)+\bar{N}(r, \infty ; f)-N_{0}\left(r, 0 ; f^{\prime}\right)+S(r, f)$
(ii) $\bar{N}_{g>1}(r, 1 ; f) \leq \bar{N}(r, 0 ; g)+\bar{N}(r, \infty ; g)-N_{0}\left(r, 0 ; g^{\prime}\right)+S(r, g)$.

\section{Proof of the Theorem}

Proof of Theorem 1. Let $F=\frac{\left[f^{n} P(f)\right]^{(k)}}{p}$ and $G=\frac{\left[g^{n} P(g)\right]^{(k)}}{p}$, where $P(w)=$ $(w-1)^{m}$. It follows that $F$ and $G$ share $\left(1, k_{1}\right)$ except for the zeros of $p(z)$.

Case 1 Let $\mathrm{H} \not \equiv 0$.

Subcase $1.1 k_{1} \geq 1$.

From (1) it can be easily calculated that the possible poles of $\mathrm{H}$ occur at (i) multiple zeros of $F$ and $G$, (ii) those 1 points of $F$ and $G$ whose multiplicities are different, (iii) poles of $F$ and $G$, (iv) zeros of $F^{\prime}\left(G^{\prime}\right)$ which are not the zeros of $F(F-1)(G(G-1))$.

Since $\mathrm{H}$ has only simple poles we get

$$
\begin{aligned}
\mathrm{N}(\mathrm{r}, \infty ; \mathrm{H}) & \leq \overline{\mathrm{N}}(\mathrm{r}, \infty ; f)+\overline{\mathrm{N}}(\mathrm{r}, \infty ; g)+\overline{\mathrm{N}}_{*}(\mathrm{r}, 1 ; \mathrm{F}, \mathrm{G})+\overline{\mathrm{N}}(\mathrm{r}, 0 ; \mathrm{F} \mid \geq 2) \\
& +\overline{\mathrm{N}}(\mathrm{r}, 0 ; \mathrm{G} \mid \geq 2)+\overline{\mathrm{N}}_{0}\left(\mathrm{r}, 0 ; \mathrm{F}^{\prime}\right)+\overline{\mathrm{N}}_{0}\left(r, 0 ; \mathrm{G}^{\prime}\right),
\end{aligned}
$$

where $\bar{N}_{0}\left(r, 0 ; F^{\prime}\right)$ is the reduced counting function of those zeros of $F^{\prime}$ which are not the zeros of $F(F-1)$ and $\bar{N}_{0}\left(r, 0 ; G^{\prime}\right)$ is similarly defined.

Let $z_{0}$ be a simple zero of $F-1$ but $p\left(z_{0}\right) \neq 0$. Then $z_{0}$ is a simple zero of $\mathrm{G}-1$ and a zero of $\mathrm{H}$. So

$$
N(r, 1 ; F \mid=1) \leq N(r, 0 ; H) \leq N(r, \infty ; H)+S(r, f)+S(r, g)
$$

While $k_{1} \geq 2$, using (35) and (36) we get

$$
\begin{aligned}
& \bar{N}(r, 1 ; F) \\
& \leq N(r, 1 ; F \mid=1)+\bar{N}(r, 1 ; F \mid \geq 2) \leq \bar{N}(r, \infty ; f) \\
& \quad+\bar{N}(r, \infty ; g)+\bar{N}(r, 0 ; F \mid \geq 2)+\bar{N}(r, 0 ; G \mid \geq 2)+\bar{N}_{*}(r, 1 ; F, G) \\
& \quad+\bar{N}(r, 1 ; F \mid \geq 2)+\bar{N}_{0}\left(r, 0 ; F^{\prime}\right)+\bar{N}_{0}\left(r, 0 ; G^{\prime}\right)+S(r, f)+S(r, g) .
\end{aligned}
$$


Now in view of Lemma 3 we get

$$
\begin{aligned}
& \bar{N}_{0}\left(r, 0 ; G^{\prime}\right)+\bar{N}(r, 1 ; F \mid \geq 2)+\bar{N}_{*}(r, 1 ; F, G) \\
& \leq \bar{N}_{0}\left(r, 0 ; G^{\prime}\right)+\bar{N}(r, 1 ; F \mid \geq 2)+\bar{N}(r, 1 ; F \mid \geq 3) \\
& \quad=\bar{N}_{0}\left(r, 0 ; G^{\prime}\right)+\bar{N}(r, 1 ; G \mid \geq 2)+\bar{N}(r, 1 ; G \mid \geq 3) \\
& \leq \bar{N}_{0}\left(r, 0 ; G^{\prime}\right)+N(r, 1 ; G)-\bar{N}(r, 1 ; G) \\
& \leq N\left(r, 0 ; G^{\prime} \mid G \neq 0\right) \leq \bar{N}(r, 0 ; G)+\bar{N}(r, \infty ; g)+S(r, g),
\end{aligned}
$$

Hence using (37), (38), Lemmas 1 and 2 we get from second fundamental theorem that

$$
\begin{aligned}
&(n+m) T(r, f) \\
& \leq T(r, F)+N_{k+2}\left(r, 0 ; f^{n} P(f)\right)-N_{2}(r, 0 ; F)+S(r, f) \\
& \leq \bar{N}(r, 0 ; F)+\bar{N}(r, \infty ; F)+\bar{N}(r, 1 ; F)+N_{k+2}\left(r, 0 ; f^{n} P(f)\right)-N_{2}(r, 0 ; F) \\
&-N_{0}\left(r, 0 ; F^{\prime}\right) \\
& \leq 2 \bar{N}(r, \infty, f)+\bar{N}(r, \infty ; g)+\bar{N}(r, 0 ; F)+N_{k+2}\left(r, 0 ; f^{n} P(f)\right) \\
&+\bar{N}(r, 0 ; F \mid \geq 2)+\bar{N}(r, 0 ; G \mid \geq 2)+\bar{N}(r, 1 ; F \mid \geq 2)+\bar{N}_{*}(r, 1 ; F, G) \\
&+\bar{N}_{0}\left(r, 0 ; G^{\prime}\right)-N_{2}(r, 0 ; F)+S(r, f)+S(r, g) \\
& \leq 2\{\bar{N}(r, \infty ; f)+\bar{N}(r, \infty ; g)\}+N_{k+2}\left(r, 0 ; f^{n} P(f)\right)+N_{2}(r, 0 ; G) \\
&+S(r, f)+S(r, g) \\
& \leq 2\{\bar{N}(r, \infty ; f)+\bar{N}(r, \infty ; g)\}+N_{k+2}\left(r, 0 ; f^{n} P(f)\right)+k \bar{N}(r, \infty ; g) \\
&+N N_{k+2}\left(r, 0 ; g^{n} P(g)\right)+S(r, f)+S(r, g) \\
& \leq 2\{\bar{N}(r, \infty ; f)+\bar{N}(r, \infty ; g)\}+(k+2) \bar{N}(r, 0 ; f)+T(r, P(f)) \\
&+(k+2) \bar{N}(r, 0 ; g)+T(r, P(g))+k \bar{N}(r, \infty ; g)+S(r, f)+S(r, g) \\
& \leq(k+4+m) T(r, f)+(2 k+4+m) T(r, g)+S(r, f)+S(r, g) \\
& \leq(3 k+8+2 m) T(r)+S(r) .
\end{aligned}
$$

In a similar way we can obtain

$$
(n+m) T(r, g) \leq(3 k+8+2 m) T(r)+S(r) .
$$

Combining (39) and (40) we see that

$$
(n+m) T(r) \leq(3 k+8+2 m) T(r)+S(r),
$$

i.e.,

$$
(n-3 k-8-m) T(r) \leq S(r) .
$$


Since $n>3 k+8+m$, (41) leads to a contradiction.

While $k_{1}=1$, using Lemmas $3,14,15,(35)$ and (36) we get

$$
\begin{aligned}
& \bar{N}(r, 1 ; F) \\
& \leq \mathrm{N}(\mathrm{r}, 1 ; \mathrm{F} \mid=1)+\overline{\mathrm{N}}_{\mathrm{L}}(\mathrm{r}, 1 ; \mathrm{F})+\overline{\mathrm{N}}_{\mathrm{L}}(\mathrm{r}, 1 ; \mathrm{G})+\overline{\mathrm{N}}_{\mathrm{E}}^{(2}(\mathrm{r}, 1 ; \mathrm{F}) \\
& \leq \overline{\mathrm{N}}(\mathrm{r}, \infty ; f)+\overline{\mathrm{N}}(\mathrm{r}, \infty ; \mathrm{g})+\overline{\mathrm{N}}(\mathrm{r}, 0 ; \mathrm{F} \mid \geq 2)+\overline{\mathrm{N}}(\mathrm{r}, 0 ; \mathrm{G} \mid \geq 2) \\
& +\bar{N}_{*}(r, 1 ; F, G)+\bar{N}_{L}(r, 1 ; F)+\bar{N}_{L}(r, 1 ; G)+\bar{N}_{E}^{(2}(r, 1 ; F)+\bar{N}_{0}\left(r, 0 ; F^{\prime}\right) \\
& +\bar{N}_{0}\left(r, 0 ; G^{\prime}\right)+S(r, f)+S(r, g) \\
& \leq \overline{\mathrm{N}}(\mathrm{r}, \infty ; f)+\overline{\mathrm{N}}(\mathrm{r}, \infty ; \mathrm{g})+\overline{\mathrm{N}}(\mathrm{r}, 0 ; \mathrm{F} \mid \geq 2)+\overline{\mathrm{N}}(\mathrm{r}, 0 ; \mathrm{G} \mid \geq 2) \\
& +2 \bar{N}_{\mathrm{L}}(\mathrm{r}, 1 ; \mathrm{F})+2 \overline{\mathrm{N}}_{\mathrm{L}}(\mathrm{r}, 1 ; \mathrm{G})+\overline{\mathrm{N}}_{\mathrm{E}}^{(2}(\mathrm{r}, 1 ; \mathrm{F})+\overline{\mathrm{N}}_{0}\left(\mathrm{r}, 0 ; \mathrm{F}^{\prime}\right) \\
& +\overline{\mathrm{N}}_{0}\left(\mathrm{r}, 0 ; \mathrm{G}^{\prime}\right)+\mathrm{S}(\mathrm{r}, \mathrm{f})+\mathrm{S}(\mathrm{r}, \mathrm{g}) \\
& \leq \overline{\mathrm{N}}(\mathrm{r}, \infty ; f)+\overline{\mathrm{N}}(\mathrm{r}, \infty ; g)+\overline{\mathrm{N}}(\mathrm{r}, 0 ; \mathrm{F} \mid \geq 2)+\overline{\mathrm{N}}(\mathrm{r}, 0 ; \mathrm{G} \mid \geq 2) \\
& +\bar{N}_{F>2}(r, 1 ; G)+N(r, 1 ; G)-\bar{N}(r, 1 ; G)+\bar{N}_{0}\left(r, 0 ; F^{\prime}\right)+\bar{N}_{0}\left(r, 0 ; G^{\prime}\right) \\
& +\mathrm{S}(\mathrm{r}, \mathrm{f})+\mathrm{S}(\mathrm{r}, \mathrm{g}) \\
& \leq \frac{3}{2} \overline{\mathrm{N}}(\mathrm{r}, \infty ; f)+\overline{\mathrm{N}}(\mathrm{r}, \infty ; g)+\overline{\mathrm{N}}(\mathrm{r}, 0 ; \mathrm{F} \mid \geq 2)+\frac{1}{2} \overline{\mathrm{N}}(\mathrm{r}, 0 ; \mathrm{F}) \\
& +\bar{N}(r, 0 ; G \mid \geq 2)+N(r, 1 ; G)-\bar{N}(r, 1 ; G)+\bar{N}_{0}\left(r, 0 ; G^{\prime}\right) \\
& +\overline{\mathrm{N}}_{0}\left(r, 0 ; F^{\prime}\right)+S(r, f)+S(r, g) \\
& \leq \frac{3}{2} \overline{\mathrm{N}}(\mathrm{r}, \infty ; f)+\overline{\mathrm{N}}(\mathrm{r}, \infty ; g)+\overline{\mathrm{N}}(\mathrm{r}, 0 ; \mathrm{F} \mid \geq 2)+\frac{1}{2} \overline{\mathrm{N}}(\mathrm{r}, 0 ; \mathrm{F}) \\
& +\bar{N}(r, 0 ; G \mid \geq 2)+N\left(r, 0 ; G^{\prime} \mid G \neq 0\right)+\bar{N}_{0}\left(r, 0 ; F^{\prime}\right)+S(r, f)+S(r, g) \\
& \leq \frac{3}{2} \overline{\mathrm{N}}(\mathrm{r}, \infty ; f)+2 \overline{\mathrm{N}}(\mathrm{r}, \infty ; g)+\overline{\mathrm{N}}(\mathrm{r}, 0 ; \mathrm{Fl} \geq 2)+\frac{1}{2} \overline{\mathrm{N}}(\mathrm{r}, 0 ; \mathrm{F}) \\
& +\mathrm{N}_{2}(r, 0 ; G)+\bar{N}_{0}\left(r, 0 ; F^{\prime}\right)+S(r, f)+S(r, g) \text {. }
\end{aligned}
$$

Hence using (42), Lemmas 1 and 2 we get from second fundamental theorem that

$$
\begin{aligned}
& (n+m) T(r, f) \\
& \leq T(r, F)+N_{k+2}\left(r, 0 ; f^{n} P(f)\right)-N_{2}(r, 0 ; F)+S(r, f) \\
& \leq \bar{N}(r, 0 ; F)+\bar{N}(r, \infty ; F)+\bar{N}(r, 1 ; F)+N_{k+2}\left(r, 0 ; f^{n} P(f)\right)-N_{2}(r, 0 ; F) \\
& \quad-N_{0}\left(r, 0 ; F^{\prime}\right) \\
& \leq \frac{5}{2} \bar{N}(r, \infty, f)+2 \bar{N}(r, \infty ; g)+N_{2}(r, 0 ; F)+\frac{1}{2} \bar{N}(r, 0 ; F)
\end{aligned}
$$




$$
\begin{aligned}
& +N_{k+2}\left(r, 0 ; f^{n} P(f)\right)+N_{2}(r, 0 ; G)-N_{2}(r, 0 ; F)+S(r, f)+S(r, g) \\
\leq & \frac{5}{2} \bar{N}(r, \infty ; f)+2 \bar{N}(r, \infty ; g)+N_{k+2}\left(r, 0 ; f^{n} P(f)\right)+\frac{1}{2} \bar{N}(r, 0 ; F) \\
& +N_{2}(r, 0 ; G)+S(r, f)+S(r, g) \\
\leq & \frac{5}{2} \bar{N}(r, \infty ; f)+2 \bar{N}(r, \infty ; g)+N_{k+2}\left(r, 0 ; f^{n} P(f)\right)+k \bar{N}(r, \infty ; g) \\
& +N_{k+2}\left(r, 0 ; g^{n} P(g)\right)+\frac{1}{2}\{k \bar{N}(r, \infty ; f) \\
& \left.+N_{k+1}\left(r, 0 ; f^{n} P(f)\right)\right\}+S(r, f)+S(r, g) \\
\leq & \frac{5+k}{2} \bar{N}(r, \infty ; f)+(k+2) \bar{N}(r, \infty ; g)+\frac{3 k+5}{2} \bar{N}(r, 0 ; f) \\
& +\frac{3}{2} T(r, P(f))+(k+2) \bar{N}(r, 0 ; g)+T(r, P(g))+S(r, f)+S(r, g) \\
\leq & \left(2 k+5+\frac{3 m}{2}\right) T(r, f)+(2 k+4+m) T(r, g)+S(r, f)+S(r, g) \\
\leq & \left(4 k+9+\frac{5 m}{2}\right) T(r)+S(r) .
\end{aligned}
$$

In a similar way we can obtain

$$
(n+m) T(r, g) \leq\left(4 k+9+\frac{5 m}{2}\right) T(r)+S(r) .
$$

Combining (43) and (44) we see that

$$
\left(n-4 k-9-\frac{3 m}{2}\right) T(r) \leq S(r) .
$$

Since $n>4 k+9+\frac{3 m}{2},(45)$ leads to a contradiction.

Subcase $1.2 k_{1}=0$. Here (36) changes to

$$
N_{E}^{1)}(r, 1 ; F \mid=1) \leq N(r, 0 ; H) \leq N(r, \infty ; H)+S(r, F)+S(r, G) .
$$

Using Lemmas 3, 16, 17, 18, (35) and (46) we get

$$
\begin{aligned}
& \bar{N}(r, 1 ; F) \\
& \leq N_{E}^{1)}(r, 1 ; F)+\bar{N}_{L}(r, 1 ; F)+\bar{N}_{L}(r, 1 ; G)+\bar{N}_{E}^{(2}(r, 1 ; F) \\
& \leq \\
& \quad \bar{N}(r, \infty ; f)+\bar{N}(r, \infty ; g)+\bar{N}(r, 0 ; F \mid \geq 2)+\bar{N}(r, 0 ; G \mid \geq 2) \\
& \quad+\bar{N}_{*}(r, 1 ; F, G)+\bar{N}_{L}(r, 1 ; F)+\bar{N}_{L}(r, 1 ; G)+\bar{N}_{E}^{(2}(r, 1 ; F)+\bar{N}_{0}\left(r, 0 ; F^{\prime}\right)
\end{aligned}
$$




$$
\begin{aligned}
& +\bar{N}_{0}\left(r, 0 ; G^{\prime}\right)+S(r, f)+S(r, g) \\
& \leq \overline{\mathrm{N}}(r, \infty ; f)+\overline{\mathrm{N}}(r, \infty ; g)+\overline{\mathrm{N}}(r, 0 ; \mathrm{F} \mid \geq 2)+\overline{\mathrm{N}}(r, 0 ; \mathrm{G} \mid \geq 2) \\
& +2 \overline{\mathrm{N}}_{\mathrm{L}}(\mathrm{r}, 1 ; \mathrm{F})+2 \overline{\mathrm{N}}_{\mathrm{L}}(\mathrm{r}, 1 ; \mathrm{G})+\overline{\mathrm{N}}_{\mathrm{E}}^{(2}(\mathrm{r}, 1 ; \mathrm{F}) \\
& +\bar{N}_{0}\left(r, 0 ; F^{\prime}\right)+\bar{N}_{0}\left(r, 0 ; G^{\prime}\right)+S(r, f)+S(r, g) \\
& \leq \overline{\mathrm{N}}(r, \infty ; f)+\overline{\mathrm{N}}(r, \infty ; g)+\overline{\mathrm{N}}(r, 0 ; \mathrm{F} \mid \geq 2)+\overline{\mathrm{N}}(r, 0 ; \mathrm{G} \mid \geq 2) \\
& +\bar{N}_{F>1}(r, 1 ; G)+\bar{N}_{G>1}(r, 1 ; F)+\bar{N}_{L}(r, 1 ; F)+N(r, 1 ; G)-\bar{N}(r, 1 ; G) \\
& +\bar{N}_{0}\left(r, 0 ; F^{\prime}\right)+\bar{N}_{0}\left(r, 0 ; G^{\prime}\right)+S(r, f)+S(r, g) \\
& \leq 3 \overline{\mathrm{N}}(\mathrm{r}, \infty ; f)+2 \overline{\mathrm{N}}(\mathrm{r}, \infty ; g)+\mathrm{N}_{2}(\mathrm{r}, 0 ; \mathrm{F})+\overline{\mathrm{N}}(\mathrm{r}, 0 ; \mathrm{F})+\mathrm{N}_{2}(\mathrm{r}, 0 ; \mathrm{G}) \\
& +N(r, 1 ; G)-\bar{N}(r, 1 ; G)+\bar{N}_{0}\left(r, 0 ; G^{\prime}\right)+\bar{N}_{0}\left(r, 0 ; F^{\prime}\right) \\
& +S(r, f)+S(r, g) \\
& \leq 3 \overline{\mathrm{N}}(\mathrm{r}, \infty ; f)+2 \overline{\mathrm{N}}(\mathrm{r}, \infty ; g)+\mathrm{N}_{2}(\mathrm{r}, 0 ; \mathrm{F})+\overline{\mathrm{N}}(\mathrm{r}, 0 ; \mathrm{F})+\mathrm{N}_{2}(\mathrm{r}, 0 ; \mathrm{G}) \\
& +N\left(r, 0 ; G^{\prime} \mid G \neq 0\right)+\bar{N}_{0}\left(r, 0 ; F^{\prime}\right)+S(r, f)+S(r, g) \\
& \leq 3 \overline{\mathrm{N}}(r, \infty ; f)+3 \overline{\mathrm{N}}(\mathrm{r}, \infty ; g)+\mathrm{N}_{2}(\mathrm{r}, 0 ; \mathrm{F})+\overline{\mathrm{N}}(\mathrm{r}, 0 ; \mathrm{F})+\mathrm{N}_{2}(\mathrm{r}, 0 ; \mathrm{G}) \\
& +\bar{N}(r, 0 ; G)+\bar{N}_{0}\left(r, 0 ; F^{\prime}\right)+S(r, f)+S(r, g) \text {. }
\end{aligned}
$$

Hence using (47), Lemmas 1 and 2 we get from second fundamental theorem that

$$
\begin{aligned}
&(n+m) T(r, f) \\
& \leq T(r, F)+N_{k+2}\left(r, 0 ; f^{n} P(f)\right)-N_{2}(r, 0 ; F)+S(r, f) \\
& \leq \bar{N}(r, 0 ; F)+\bar{N}(r, \infty ; F)+\bar{N}(r, 1 ; F)+N_{k+2}\left(r, 0 ; f^{n} P(f)\right)-N_{2}(r, 0 ; F) \\
&-N_{0}\left(r, 0 ; F^{\prime}\right) \\
& \leq 4 \bar{N}(r, \infty, f)+3 \bar{N}(r, \infty ; g)+N_{2}(r, 0 ; F)+2 \bar{N}(r, 0 ; F) \\
&+N_{k+2}\left(r, 0 ; f^{n} P(f)\right)+N_{2}(r, 0 ; G)+\bar{N}(r, 0 ; G)-N_{2}(r, 0 ; F) \\
&+S(r, f)+S(r, g) \\
& \leq 4 \bar{N}(r, \infty ; f)+3 \bar{N}(r, \infty ; g)+N_{k+2}\left(r, 0 ; f^{n} P(f)\right)+2 \bar{N}(r, 0 ; F) \\
&+N(r, 0 ; G)+\bar{N}(r, 0 ; G)+S(r, f)+S(r, g) \\
& \leq 4 \bar{N}(r, \infty ; f)+3 \bar{N}(r, \infty ; g)+N_{k+2}\left(r, 0 ; f^{n} P(f)\right)+2 k \bar{N}(r, \infty ; f) \\
&+2 N_{k+1}\left(r, 0 ; f^{n} P(f)\right)+k \bar{N}(r, \infty ; g)+N_{k+2}\left(r, 0 ; g^{n} P(g)\right) \\
&+k \bar{N}(r, \infty ; g)+N N_{k+1}\left(r, 0 ; g^{n} P(g)\right)+S(r, f)+S(r, g)
\end{aligned}
$$




$$
\begin{aligned}
\leq & (2 k+4) \bar{N}(r, \infty ; f)+(2 k+3) \bar{N}(r, \infty ; g)+(3 k+4) \bar{N}(r, 0 ; f) \\
& +3 T(r, P(f))+(2 k+3) \bar{N}(r, 0 ; g)+2 T(r, P(g))+S(r, f)+S(r, g) \\
\leq & (5 k+8+3 m) T(r, f)+(4 k+6+2 m) T(r, g)+S(r, f)+S(r, g) \\
\leq & (9 k+14+5 m) T(r)+S(r) .
\end{aligned}
$$

In a similar way we can obtain

$$
(n+m) T(r, g) \leq(9 k+14+5 m) T(r)+S(r) .
$$

Combining (48) and (49) we see that

$$
(n-9 k-14-4 m) T(r) \leq S(r) .
$$

Since $n>9 k+14+4 m,(50)$ leads to a contradiction.

Case 2. Let $\mathrm{H} \equiv 0$. Then by Lemma 10 we get either

$$
f^{n}(f-1)^{m} \equiv g^{n}(g-1)^{m}
$$

or

$$
\left[f^{n}(f-1)^{m}\right]^{(k)}\left[g^{n}(g-1)^{m}\right]^{(k)} \equiv p^{2} .
$$

We now consider following two subcases.

Subcase 2.1: Let $m=0$.

Now from (51) we get $f^{n} \equiv g^{n}$ and so $f \equiv t g$, where $t$ is a constant satisfying $t^{n}=1$.

Also from (52) we get

$$
\left[f^{n}\right]^{(k)}\left[g^{n}\right]^{(k)} \equiv p^{2}
$$

Then by Lemma 11 we get the conclusion (1).

Subcase 2.2: Let $m \geq 1$.

Applying Lemma 13, from (52) we see that

$$
\left[f^{n}(f-1)^{m}\right]^{(k)}\left[g^{n}(g-1)^{m}\right]^{(k)} \not \equiv p^{2},
$$

for $k=1$.

In addition, when $f$ and $g$ share $(\infty, 0)$, then by Lemma 12 we must have

$$
\left[f^{n}(f-1)^{m}\right]^{(k)}\left[g^{n}(g-1)^{m}\right]^{(k)} \not \equiv p^{2} .
$$

Next we consider the relation (51) and let $h=\frac{g}{f}$. 
First we suppose that $h$ is non-constant.

For $m=1$ : Then from (51) we get $f \equiv \frac{1-h^{\mathfrak{n}}}{1-h^{n+1}}$, i.e.,

$$
f \equiv\left(\frac{h^{n}}{1+h+h^{2}+\ldots+h^{n}}-1\right) .
$$

Hence by Lemma 1 we get

$$
T(r, f)=T\left(r, \sum_{j=0}^{n} \frac{1}{h^{j}}\right)+O(1)=n T\left(r, \frac{1}{h}\right)+S(r, h)=n T(r, h)+S(r, h) .
$$

Similarly we have $T(r, g)=n T(r, h)+S(r, h)$. Therefore $S(r, f)=S(r, g)=$ $S(r, h)$.

Also it is clear that

$$
\sum_{j=1}^{n} \bar{N}\left(r, u_{j} ; h\right) \leq \bar{N}(r, \infty ; f)
$$

where $u_{j}=\exp \left(\frac{2 j \pi i}{n+1}\right)$ and $j=1,2, \ldots, n$.

Then by the second fundamental theorem we get

$$
(n-2) T(r, h) \leq \sum_{j=1}^{n} \bar{N}\left(r, u_{j} ; h\right)+S(r, f) \leq \bar{N}(r, \infty ; f)+S(r, f) .
$$

Similarly we have

$$
(n-2) T(r, h) \leq \bar{N}(r, \infty ; g)+S(r, g) .
$$

Adding and simplifying these we get

$$
2(n-2) T(r, h) \leq n(2-\Theta(\infty ; f)-\Theta(\infty ; g)+\varepsilon) T(r, h)+S(r, h),
$$

where $0<\varepsilon<\Theta(\infty ; f)+\Theta(\infty ; g)$. This leads to a contradiction as $\Theta(\infty ; f)+$ $\Theta(\infty ; g)>\frac{4}{n}$.

For $m \geq 2$ : Then from (51) we can say that $f$ and $g$ satisfying the algebraic equation $R(f, g)=0$, where

$$
R\left(\omega_{1}, \omega_{2}\right)=\omega_{1}^{n}\left(\omega_{1}-1\right)^{m}-\omega_{2}^{n}\left(\omega_{2}-1\right)^{m} .
$$

Next we suppose that $h$ is a constant.

Then from (51) we get

$$
f^{n} \sum_{i=0}^{m}(-1)^{i}{ }^{m} C_{m-i} f^{m-i} \equiv g^{n} \sum_{i=0}^{m}(-1)^{i}{ }^{m} C_{m-i} g^{m-i} .
$$


Now substituting $\mathrm{g}=\mathrm{fh}$ in (53) we get

$$
\sum_{i=0}^{m}(-1)^{i}{ }^{m} C_{m-i} f^{n+m-i}\left(h^{n+m-i}-1\right) \equiv 0,
$$

which implies that $h=1$. Hence $f \equiv g$. This completes the proof.

\section{Acknowledgement}

The author is grateful to the referee for his/her valuable comments and suggestions to-wards the improvement of the paper.

\section{References}

[1] T. C. Alzahary, H. X. Yi, Weighted value sharing and a question of I. Lahiri, Complex Var. Theory Appl., 49 (15) (2004), 1063-1078.

[2] A. Banerjee, Meromorphic functions sharing one value, Int. J. Math. Math. Sci., 22 (2005), 3587-3598.

[3] M. L. Fang, X. H. Hua, Entire functions that share one value, J. Nanjing Univ. Math. Biquarterly, 13 (1) (1996), 44-48.

[4] M. L. Fang, Uniqueness and value-sharing of entire functions, Comput. Math. Appl., 44 (2002), 823-831.

[5] G. Frank, Eine Vermutung Von Hayman über Nullslellen meromorphic Funktion, Math. Z., 149 (1976), 29-36.

[6] W. K. Hayman, Meromorphic Functions, The Clarendon Press, Oxford (1964).

[7] I. Lahiri, Weighted sharing and uniqueness of meromorphic functions, Nagoya Math. J., 161 (2001), 193-206.

[8] I. Lahiri, Weighted value sharing and uniqueness of meromorphic functions, Complex Var. Theory Appl., 46 (2001), 241-253.

[9] I. Lahiri, S. Dewan, Value distribution of the product of a meromorphic function and its derivative, Kodai Math. J., 26 (2003), 95-100. 
[10] I. Lahiri, A. Sarkar, Nonlinear differential polynomials sharing 1-points with weight two, Chinese J. Contemp. Math., 25 (3) (2004), 325-334.

[11] P. Li, C. C. Yang, On the characteristics of meromorphic functions that share three values CM, J. Math. Anal. Appl., 220 (1998), 132-145.

[12] L. Liu, Uniqueness of meromorphic functions and differential polynomials, Comput. Math. Appl., 56 (2008), 3236-3245.

[13] R. Nevanlinna, Einige Eindeutigkeitssätze in der Theorie der Meromorphen Funktionen, Acta Math., 48 (1926), 367-391.

[14] P. Sahoo, Uniqueness and weighted value sharing of meromorphic functions, Applied. Math. E-Notes., 11 (2011), 23-32.

[15] C. C. Yang, On deficiencies of differential polynomials II, Math. Z., 125 (1972), 107-112.

[16] C. C. Yang, X. H. Hua, Uniqueness and value sharing of meromorphic functions, Ann. Acad. Sci. Fenn. Math., 22 (1997), 395-406.

[17] K. Yamanoi, The second main theorem for small functions and related problems, Acta Math., 192 (2004), 225-294.

[18] H. X. Yi, On characteristic function of a meromorphic function and its derivative, Indian J. Math., 33 (2)(1991), 119-133.

[19] H. X. Yi, C. C. Yang, Uniqueness Theory of meromorphic functions, Science Press, Beijing, 1995.

[20] Q. C. Zhang, Meromorphic function that shares one small function with its derivative, J. Inequal. Pure Appl. Math., 6 (4)(2005), Art.116 [ONLINE http://jipam.vu.edu.au/\.

[21] X. Y. Zhang, W. C. Lin, Uniqueness and value sharing of entire functions, J. Math. Anal. Appl., 343 (2008), 938-950. 